\title{
Intensity Change of Binary Tropical Cyclones (TCs) in Idealized Numerical Simulations: Two Initially Identical Mature TCs
}

\author{
HAO-YAN LiU, ${ }^{\text {a,b,c }}$ YUQING WANG, ${ }^{\text {de e }}$ AND JiAn-FEnG GU ${ }^{\mathrm{f}}$ \\ ${ }^{a}$ Key Laboratory of Marine Hazards Forecasting, Ministry of Natural Resources, Hohai University, Nanjing, China \\ ${ }^{\mathrm{b}}$ College of Oceanography, Hohai University, Nanjing, China \\ ${ }^{\mathrm{c}}$ State Key Laboratory of Severe Weather, Chinese Academy of Meteorological Sciences, China Meteorological Administration, \\ Beijing, China \\ ${ }^{\mathrm{d}}$ International Pacific Research Center, School of Ocean and Earth Science and Technology, University of Hawai'i at Mānoa, \\ Honolulu, Hawaii \\ ${ }^{\mathrm{e}}$ Department of Atmospheric Sciences, School of Ocean and Earth Science and Technology, University of Hawai'i at Mānoa, \\ Honolulu, Hawaii \\ ${ }^{\mathrm{f}}$ Department of Meteorology, University of Reading, Reading, United Kingdom
}

(Manuscript received 21 April 2020, in final form 29 December 2020)

\begin{abstract}
This study investigates the intensity change of binary tropical cyclones (TCs) in idealized cloud-resolving simulations. Four simulations of binary interaction between two initially identical mature TCs of about $70 \mathrm{~m} \mathrm{~s}^{-1}$ with initial separation distance varying from 480 to $840 \mathrm{~km}$ are conducted in a quiescent $f$-plane environment. Results show that two identical TCs finally merge if their initial separation distance is within $600 \mathrm{~km}$. The binary TCs presents two weakening stages (stages 1 and 3) with a quasi-steady evolution (stage 2) in between. Such intensity change of one TC is correlated with the upper-layer vertical wind shear (VWS) associated with the upper-level anticyclone (ULA) of the other TC. The potential temperature budget shows that eddy radial advection of potential temperature induced by large upper-layer VWS contributes to the weakening of the upper-level warm core and thereby the weakening of binary TCs in stage 1 . In stage 2 , the upper-layer VWS first weakens and then restrengthens with relatively weak magnitude, leading to a quasi-steady intensity evolution. In stage 3, due to the increasing upper-layer VWS, the nonmerging binary TCs weaken again until their separation distance exceeds the local Rossby radius of deformation of the ULA (about $1600 \mathrm{~km}$ ), which can serve as a dynamical critical distance within which direct interaction can occur between two TCs. In the merging cases, the binary TCs weaken prior to merging because highly asymmetric structure develops as a result of strong horizontal deformation of the inner core. However, the merged system intensifies shortly after merging.
\end{abstract}

KEYWORDS: Tropical cyclones; Hurricanes/typhoons; Numerical analysis/modeling

\section{Introduction}

When two tropical cyclones (TCs) interact with each other, errors in both track and intensity forecasts are often larger than those for a single TC (Brand 1970; Jarrell et al. 1978; Liu and Tan 2016). Fujiwhara $(1921,1923,1931)$ first demonstrated the interaction of two vortices in proximity in the tank experiments and found that the two cyclonic vortices rotated counterclockwise, moved toward each other, and finally merged (i.e., Fujiwhara effect). In the real atmosphere, binary TCs are detected by using various statistical parameters such as their intensities, relative locations, coexistence time and separation distance. The critical separation distance determining whether two TCs belong to binary TCs ranges from 1300 to $1800 \mathrm{~km}$ (Brand 1970; Dong and Neumann 1983; Wu et al. 2011; Jang and Chun 2015a; Ren et al. 2020). Within the critical separation distance, the motion of binary TCs presents mutual approaching, mutual cyclonic orbiting, or mutual escaping (Brand 1970; Dong and Neumann 1983; Lander and Holland 1993, hereafter LH93). Sometimes mutual anticyclonic rotation of binary TCs happens under the influence of the large-scale environmental flow such as the subtropical high (Dong

Corresponding author: Prof. Yuqing Wang, yuqing@hawaii.edu and Neumann 1983; LH93). Real case studies show that the weaker and smaller TC is susceptible to the stronger and larger TC in a binary system, where the former looks like moving around the latter (Wu et al. 2003; Yang et al. 2008). Moreover, during the binary interaction, filamentation and deformation occurred in the weaker and smaller TC Typhoon Alex (1998), which finally became a spiral band of the stronger and larger Typhoon Zeb (1998) on satellite images (Kuo et al. 2000).

More details relating to the motion and structure change of binary TCs have been investigated in idealized frameworks (Chang 1983; DeMaria and Chan 1984; Wang and Zhu 1989a,b; Waugh 1992; Dritschel and Waugh 1992; Ritchie and Holland 1993; Wang and Holland 1995, hereafter WH95; Falkovich et al. 1995; Khain et al. 2000; Prieto et al. 2003; Shin et al. 2006; Jang and Chun 2015b, hereafter JC15b). Several barotropic dynamical processes have been discussed in the literature. For example, the horizontal vorticity advection is key to whether the binary TCs may approach each other in nondivergent barotropic model (DeMaria and Chan 1984; Wang and Zhu 1989a,b; Shin et al. 2006), while competition between the deformation and filamentation from the opposite vortex and the restoring force of the vortex itself determines whether the two interacting vortices merge or not (Dritschel and Waugh 1992; Waugh 1992; Ritchie and Holland 1993; Prieto et al. 2003). 
The binary interaction in three-dimensional models is more complicated by resolving the baroclinic TC structure, surface friction, and diabatic heating (Chang 1983; Falkovich et al. 1995; WH95). Compared with dry simulations, diabatic heating can modulate the vortex structure in moist simulations, in which the vortices show relatively axisymmetric structure during the merging (WH95; Khain et al. 2000). WH95 found that two identical TC vortices with the initial separation distance less than $640 \mathrm{~km}$ on an $f$ plane experienced rapid mutual approaching and weakening before merging. They also indicated that the merger of binary TCs was a bottom-up process where the low-level vortices merged first, followed by the merging of the middle-level vortices with much stronger filamentation. The simulation of WH95 also demonstrated a mutual orbiting process, followed by release and escape, or merging, which was consistent with the conceptual model of binary interaction proposed by LH93. More recently, JC15b demonstrated that greater environmental convective available potential energy and higher maximum potential intensity could lead to stronger binary interaction, while the beta effect could weaken the mutual approaching of binary TCs by generating asymmetric structure.

Although previous studies have revealed many features of binary-TCs interaction, most of them focused on the track characteristics of two interacting vortices. Limited studies have investigated the intensity changes of binary TCs. In addition, the coarse model resolution and the use of cumulus parameterization in previous studies are not adequate for understanding the intensity change of binary TCs (e.g., WH95; Khain et al. 2000; JC15b). In this study, we will examine the intensity change of two interacting TCs and understand the involved dynamical processes using an idealized three-dimensional fullphysics model. The rest of the paper is organized as follows. The numerical model and experimental design are described in section 2. Section 3 discusses the main results with the focus on the storm structure and intensity changes of the simulated binary TCs. Summary and conclusions are given in the last section.

\section{Numerical model and experimental design}

\section{a. Model setup}

In this study, the advanced Weather Research and Forecasting (WRF) Model, version 3.4 (Skamarock et al. 2008), was used to investigate the interaction between two TCs. The WRF Model is a three-dimensional nonhydrostatic, full-physics, atmospheric model. The model domain was configured with two nested interactive meshes of $901 \times 901(\mathrm{D} 01)$ and $901 \times 901(\mathrm{D} 02)$ grid points, with their horizontal grid spacings of 9 and $3 \mathrm{~km}$, respectively. The open lateral boundary condition was utilized for D01. The model atmosphere had 50 vertical levels topped at the $25-\mathrm{km}$ height (about $28 \mathrm{hPa}$ ) with 18 vertical levels below the $3-\mathrm{km}$ height. The model physics included the WRF 5-class microphysics scheme (WSM5; Hong et al. 2004) and the Yonsei University (YSU) planetary boundary layer (PBL) scheme (Hong et al. 2006). The Kain-Fritsch cumulus parameterization scheme (Kain and Fritsch 1990) was applied only in D01. The scheme for drag and enthalpy coefficients was Donelan $\mathrm{Cd}+$ Constant Ck
(Donelan et al. 2004). The radiations were not activated in this study. All experiments were performed on an $f$ plane at $20^{\circ} \mathrm{N}$ over ocean with a uniform sea surface temperature (SST) of $28^{\circ} \mathrm{C}$.

\section{b. Initial vortex}

The initial axisymmetric vortex for all experiments of binary TCs was spun up from a single TC integration (ORIG). In ORIG, the Jordan sounding (Jordan 1958) was used as the unperturbed environment. The initial vortex in ORIG had the radial profile of tangential wind following that in Fiorino and Elsberry (1989). The maximum tangential wind speed was $30 \mathrm{~m} \mathrm{~s}^{-1}$ at the radius of $120 \mathrm{~km}$ (RMW) with the exponential decaying factor of 1.0. The maximum wind decreased with height to zero at about $100 \mathrm{hPa}$ following Wang (2007). The vortex in ORIG was initially located at the center of the two meshes and spun up for $96 \mathrm{~h}$ when the storm reached its mature stage. We separated the axisymmetric vortex of the TC after the 96-h simulation as the initial vortex and used the mean fields after removing the axisymmetric vortex as the environmental fields in all binary-TCs experiments. The vortex separation followed that in Liu and Tan (2016), which was an extension of the method introduced by Cha and Wang (2013) and included the following four steps. First, a local three-point spatial filter was conducted, respectively, in the meridional and zonal directions to separate the basic field and the disturbance field. Second, a cylindrical filter was applied to further extract the vortex component from the disturbance field. Third, the axisymmetric TC vortex was calculated as the azimuthally averaged vortex component. Finally, the environmental field was obtained by spatially averaging the total field excluding the axisymmetric vortex. More details can be found in Cha and Wang (2013) and Liu and Tan (2016), and references therein. Figure 1a shows the obtained axisymmetric tangential and radial wind fields of the TC vortex after the 96-h spinup in ORIG. The RMW at the top of the boundary layer was about $60 \mathrm{~km}$ and tilted radially outward with height. The inflow was below $2 \mathrm{~km}$, and the outflow occurred mainly in the upper troposphere. The anticyclonic circulation in the upper troposphere (above the 14-km height) was outside of a radius of about $500 \mathrm{~km}$ from the TC surface center. The environmental sounding was very close to the Jordan sounding (not shown).

To ensure the validity of the initial conditions composited by the axisymmetric TC vortex and the mean environmental field in our binary-TCs experiments, we first ran a simulation using a single TC initial condition as a reference (CTRL). Figure 1b compares the intensity evolutions of the simulated TCs in ORIG and CTRL. In ORIG, the TC intensified and reached its mature stage after about 90-h simulation. In CTRL, the vortex experienced some quick adjustment and then reached a steadystage evolution as in ORIG. Although the intensity evolutions in terms of the maximum 10-m-height wind speed (Vmax) and the minimum sea level pressure (MSLP) differ in detail in CTRL and ORIG, the difference can be considered relatively small. This indicates that the axisymmetric vortex obtained from the 96-h spinup in ORIG reached its mature phase. Thus, any significant change in intensity in the binary-TCs simulations can be considered as a result of the binary interaction when two TCs vortices were simultaneously introduced. 

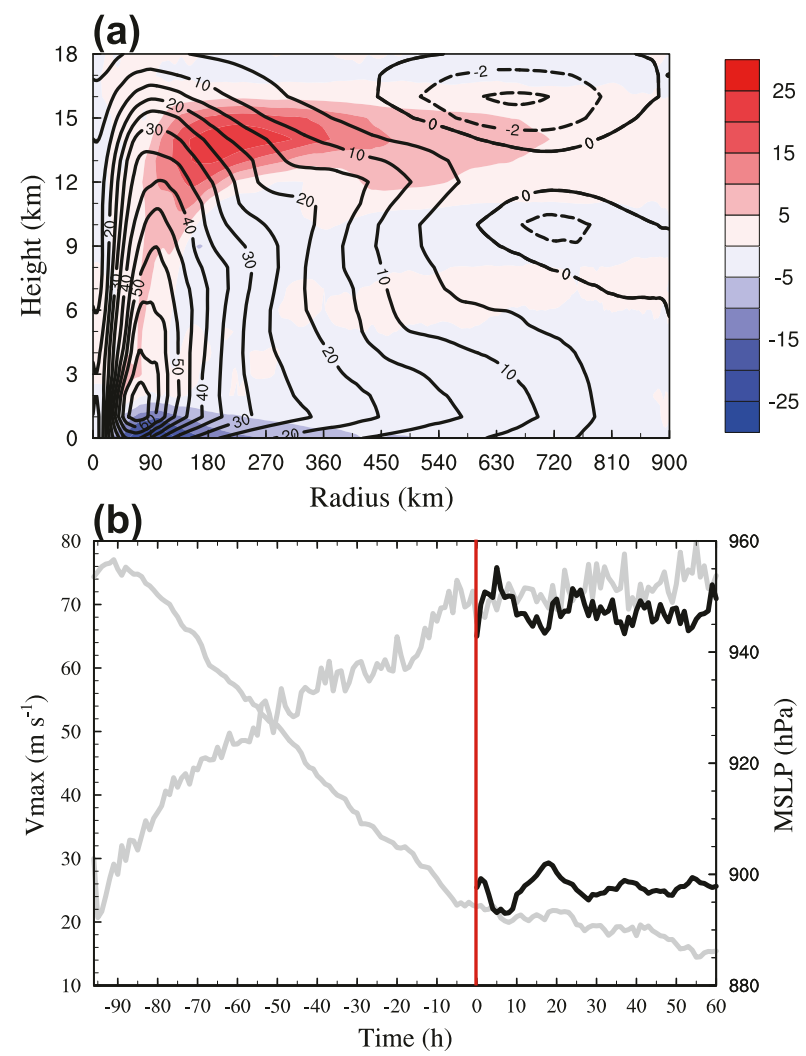

FIG. 1. (a) Radial-height distribution of the azimuthal mean radial $\left(\mathrm{m} \mathrm{s}^{-1}\right.$, interval of $5 \mathrm{~m} \mathrm{~s}^{-1}$, shading) and tangential $\left(\mathrm{m} \mathrm{s}^{-1}\right.$, contours) winds of the TC at the initial time in CTRL (after a 96-h spinup of the model TC vortex). Positive values (solid) with contour intervals of $5 \mathrm{~m} \mathrm{~s}^{-1}$ are for cyclonic circulation and negative values (dashed) with contour intervals of $2 \mathrm{~m} \mathrm{~s}^{-1}$ are for anticyclonic circulation. (b) The temporal evolutions of the $\operatorname{Vmax}\left(\mathrm{m} \mathrm{s}^{-1}\right)$ at the 10-m height and the MSLP (hPa) in ORIG (gray) and CTRL (black). The ORIG simulation started from $-96 \mathrm{~h}$ and the CTRL simulation started from $0 \mathrm{~h}$ (see more details in the text).

\section{c. Experimental design}

To investigate the interaction of binary TCs, we conducted four experiments with different initial separation distances, which were 480 (D480), 600 (D600), 720 (D720), and $840 \mathrm{~km}$ (D840), respectively. In each experiment, the two initial TCs, which were the same as that in CTRL above, were placed in the east-west direction. The centroid of the binary TCs was located at the center of D01 and D02. For the convenience of discussion, we named the WTC for the TC initially located to the west and the ETC for the TC initially located to the east.

Some parameters in the following analysis/discussions are defined here. The TC surface center was simply defined as the location of the MSLP. We will discuss the TC intensity change in terms of the change in MSLP rather than Vmax (e.g., WH95; Falkovich et al. 1995; Khain et al. 2000; JC15b) because the MSLP of one TC can be detected even when binary TCs are undergoing merging process. The eye region was roughly defined as the area from the TC surface center to a radius of $60 \mathrm{~km}$, which was the same as the initial RMW at the low levels in the binary-TCs experiments. The inner core was referred to the area from the TC surface center to a radius of $180 \mathrm{~km}$, which was about 3 times the initial RMW at the low levels (Wang 2008).

\section{Results}

\section{a. An overview}

Figure 2 shows the track and intensity evolutions of the binary TCs, together with the change of the separation distance between the centers of two TCs. For a comparison, the intensity evolution of a single TC in CTRL is also shown in Fig. 2. Both TCs mutually rotated cyclonically in all experiments. The two TCs merged as a new TC after 22 and $67 \mathrm{~h}$ in D480 and D600, respectively. In D720 and D840, the binary TCs did not merge with their separation distance increasing with time. As the separation distances increased, the cyclonic rotation also slowed down and the two TCs eventually departed from each other without significant rotation, such as the case in D840 (Fig. 2g). These results are roughly consistent with previous studies (WH95; JC15b) and the conceptual model of binaryTCs interaction in LH93.

We divided the intensity evolution into three stages (right panels in Fig. 2) depending on their interaction modes. Stage 1 is the first $10 \mathrm{~h}$ of the simulations during which the binary TCs experienced the weakening in all experiments. Stage 2 is from 10 to $55 \mathrm{~h}$ of the simulations when the intensity of the binary TCs showed little change or experienced a quasi-steady evolution in D600, D720 and D840. In stage 3, the binary TCs weakened again after $55 \mathrm{~h}$ in D600 (55-67 h), D720 (55 h to the end), and D840 (55-78 h), and prior to merging from 10 to $22 \mathrm{~h}$ of the simulation in D480. Although the binary TCs in D480 weakened during the first $22 \mathrm{~h}$ of the simulation, the physical processes that led to the two weakening periods were different. Note that an intensification of the merged system occurred in both D480 and D600, which was also found in WH95. It is interesting that the two TCs in D840 after stage 3 intensified again when the separation distance between the two TCs became larger than $1600 \mathrm{~km}$ (Fig. 2h). Since the temporal evolutions of the two TCs in the binary experiments are similar and almost symmetric with respect to their centroid due to their identical initial conditions, we take the WTC to discuss the intensity and structure changes in the following discussion.

Figure 3 shows the mean potential temperature anomaly in the eye region of the WTC in the four binary experiments. The potential temperature anomaly was calculated as the perturbation relative to the environment, which was the mean potential temperature from the TC surface center to $600-\mathrm{km}$ radius, the same as that in Fu et al. (2019). Following Zhang and Chen (2012), we also calculated the central sea level pressure rises induced by the upper-level (between the 9- and 16-km heights, $\Delta P_{u}$ ), lower-level (between the 2- and 6-km heights, $\left.\Delta P_{l}\right)$, and total-level warm core $(\Delta P)$ using the hydrostatic equation with the results shown in Fig. 3 . The bias of the central sea level pressure integrated from the hydrostatic equation and the MSLP from the model simulations was less than $5 \mathrm{hPa}$. The TC presented a warm-core structure at the initial time with two maximum positive potential temperature 
(a)

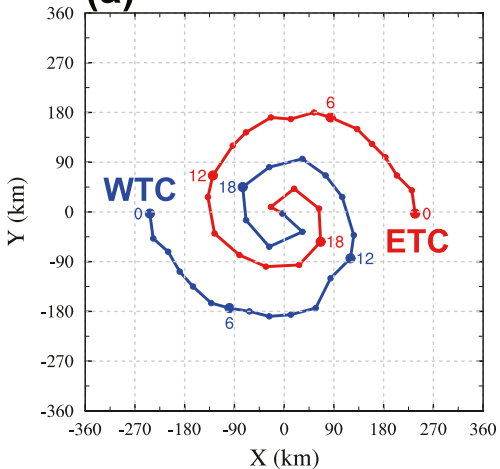

(c)

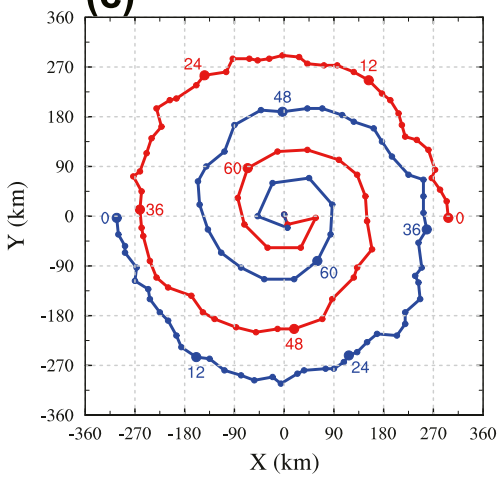

(e)

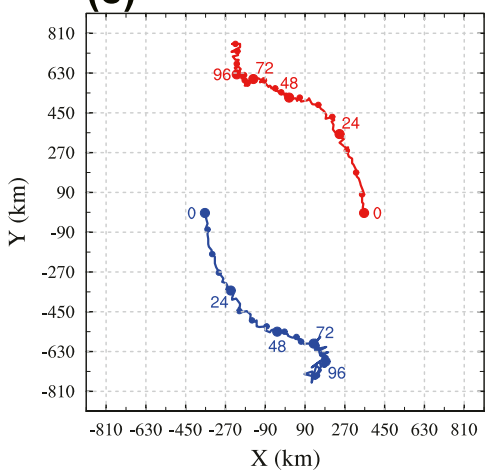

(g)

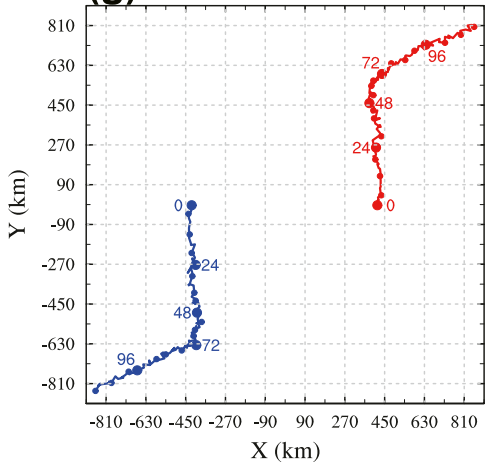

(b)

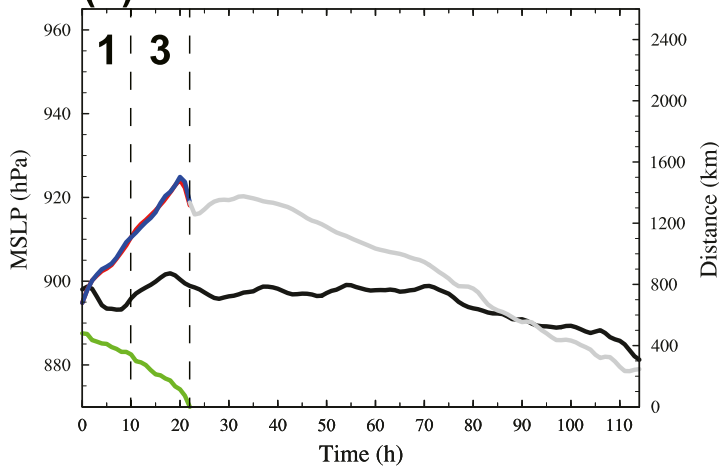

(d)

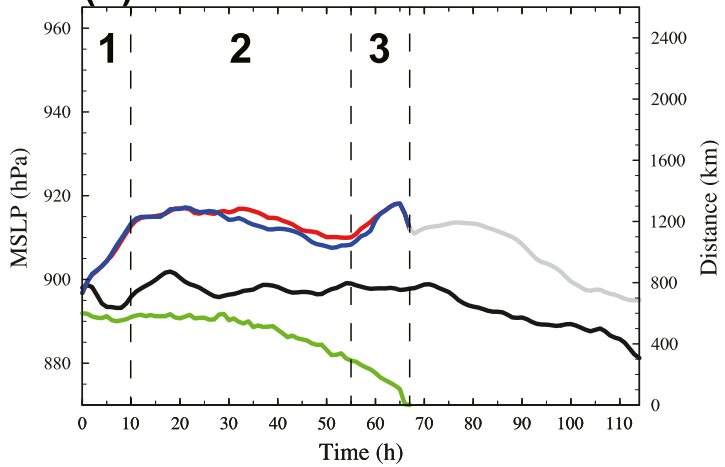

(f)

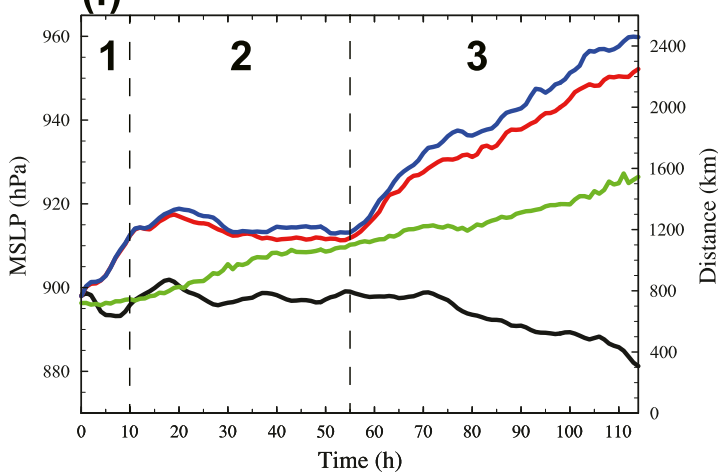

(h)

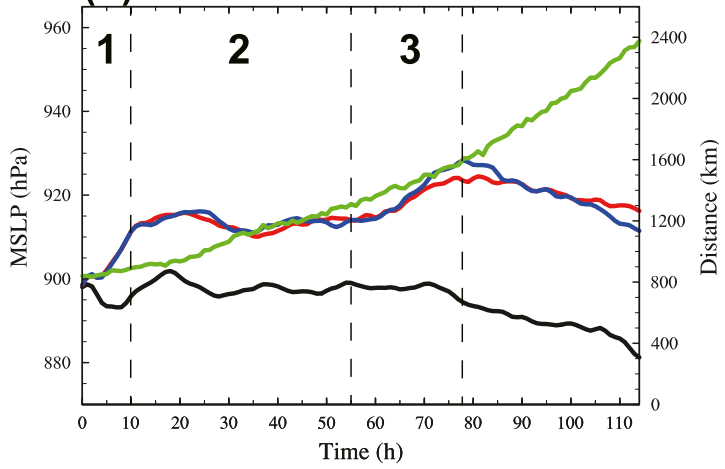

FIG. 2. (left) Tracks of the binary TCs in (a) D480, (c) D600, (e) D720, and (g) D840, respectively. The blue curves are for the WTC and the red curves are for the ETC. (right) The intensity evolutions of the WTC (blue) and the ETC (red) in (b) D480, (d) D600, (f) D720, and (h) D840, together with the intensity evolution of the TC in CTRL (black). The gray curves in (b) and (d) represent the intensity of the merged TC. The green curves denote the separation distance between the two TCs. The dashed vertical lines separate the three stages of the intensity evolution discussed in section 3. 

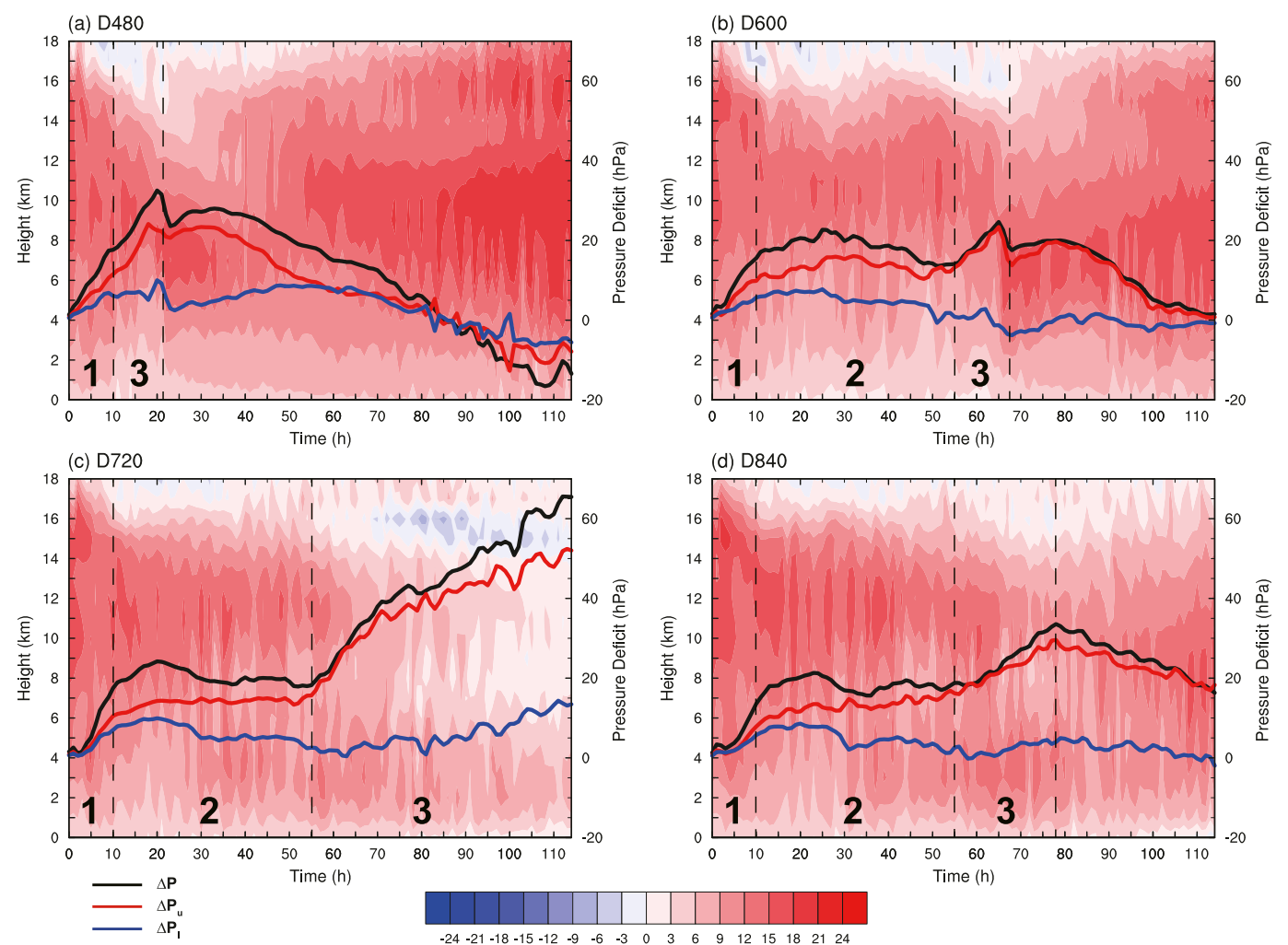

FIG. 3. Time-height cross sections of the potential temperature perturbation (K, shading) averaged within the eye region ( $r \leq 60 \mathrm{~km}$ ) of the WTC in (a) D480, (b) D600, (c) D720, and (d) D840, along with the time evolution of the central sea level pressure changes (solid line) and stage separation lines (dashed vertical lines). The central sea level pressure changes are relative to the initial time and caused by the total level ( $\Delta P$, black line), upper level $\left(\Delta P_{u}\right.$, red line), and lower level $\left(\Delta P_{l}\right.$, blue line $)$, respectively.

anomalies centered at the 10 - and $15-\mathrm{km}$ heights, respectively, in all four experiments. Influenced by the ETC, the upper-level warm core of the WTC weakened during stages 1 and 3 in all binary experiments and in the meantime both $\Delta P$ and $\Delta P_{u}$ rose. In D600, D720 and D840 (Figs. 3b-d), the upper-level warm core weakened and the lower-level warm core strengthened in stage 2, but the potential temperature change in both upper and lower levels were quite small. This can be proven by the increasing of $\Delta P_{u}$ and decreasing of $\Delta P_{l}$, and both were largely offset. As a result, $\Delta P$ did not change much in stage 2 . The warm core strengthened again as the merged TC intensified in D480 and D600 (Figs. 3a,b). After stage 3 in D840 (Fig. 3d) the warm core restrengthened with both $\Delta P$ and $\Delta P_{u}$ slowly decreased until the end of the simulation.

The above results demonstrate that the intensity change of the binary TCs is consistent with the evolution of the warmcore strength. Therefore, the key to understand the intensity change is to understand what caused the change in the warmcore strength under the binary interaction. Previous studies have demonstrated that the weakening of the TC warm core and eyewall entropy is related to the asymmetric structures in the inner-core region and associated ventilation (Simpson and Riehl 1958; Gray 1968; Frank and Ritchie 2001; Tang and Emanuel 2010; Gu et al. 2015). Figure 4 shows the evolution of the inner-core mean relative wavenumber- 1 kinetic energy
(RKE; defined as the percentage of the wavenumber-1 kinetic energy in the total kinetic energy) of the WTC. The percentages of higher wavenumbers were quite smaller than the RKE (not shown). In all experiments, the RKE was the largest at the 16-km height and smallest below the 9-km height, indicating that the upper-level (between the 9- and 16-km heights or equivalent $300-100 \mathrm{hPa}$ ) RKE was dominant. In stage 1, the RKE increased rapidly above the $14-\mathrm{km}$ height, which was consistent with the weakening of the TC in all experiments. In D600, D720, and D840, the upper-level RKE shrank in stage 2 from $10 \mathrm{~h}$ to about $30 \mathrm{~h}$ of the simulations. Then the upper-level RKE restrengthened and expanded downward. The development of upper-level RKE was prior to the increase of the MSLP, suggesting that the development of asymmetric structure in the upper troposphere was most likely responsible for the weakening of the binary TCs. In stage 3, the upper-level RKE and the MSLP increased again in all experiments. After stage 3, the RKE of the whole TC vortex decreased sharply after the two TCs merged and reintensified in D480 and D600 (Figs. 4a,b). Similar features were found after stage 3 in D840 (Fig. 4d) probably because the two TCs were far away from each other.

The dominance of wavenumber-1 asymmetry indicates that vertical wind shear (VWS) was playing an essential role in the structure change (Jones 1995; Wang and Holland 1996; 

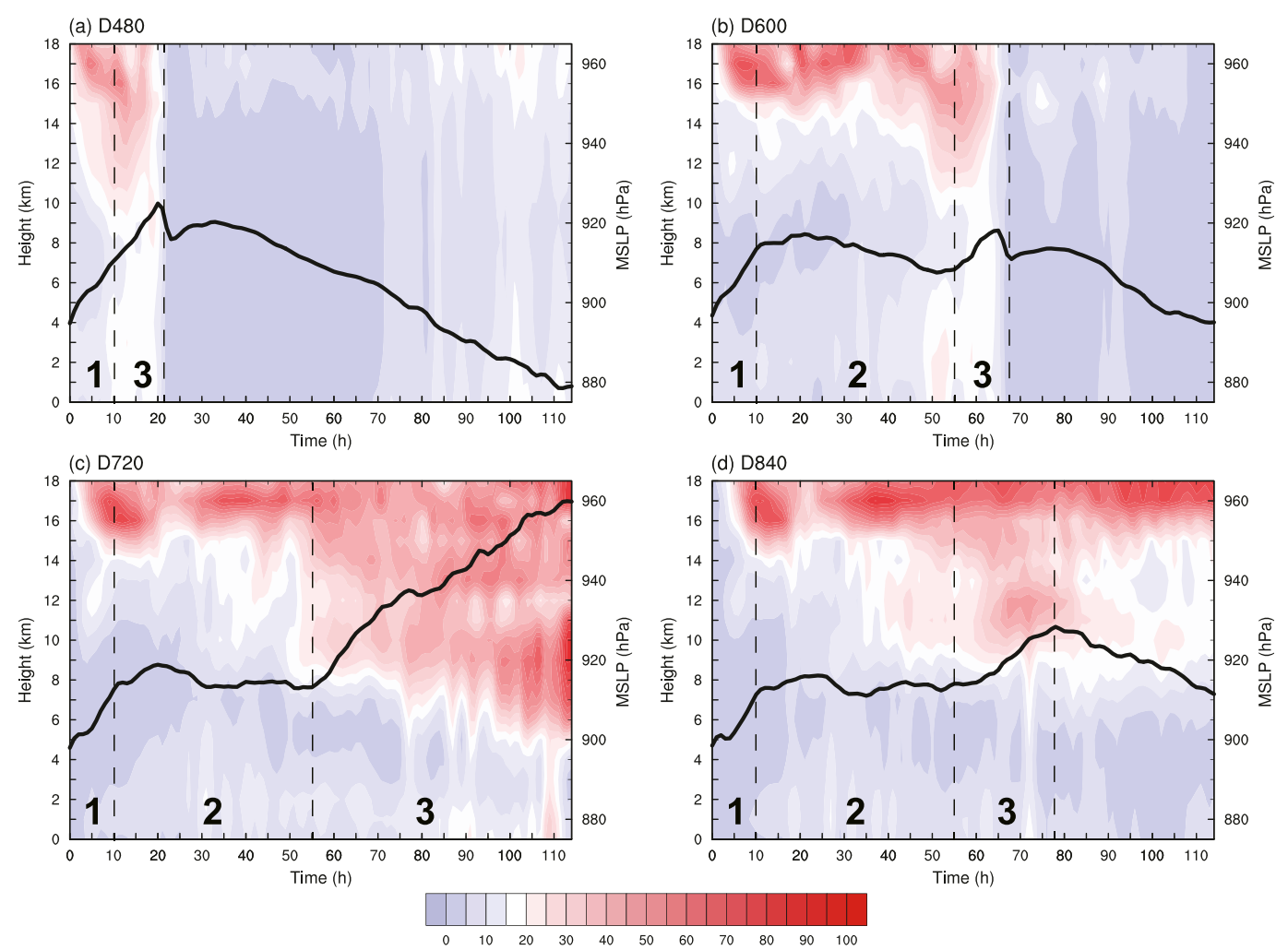

FIG. 4. Time-height cross sections of the RKE ( $\%$, shading) averaged within the inner core $(r \leq 180 \mathrm{~km})$, together with the evolution of the MSLP (solid line) and stage separation lines (dashed vertical lines).

Frank and Ritchie 2001; Corbosiero and Molinari 2003; Reasor et al. 2004; Chen et al. 2006; Xu and Wang 2013; Zhang et al. 2013; DeHart et al. 2014; Gu et al. 2016). Therefore, it is necessary to examine whether the intensity change of binary TCs is related to the asymmetric structure and the VWS. The VWS over one TC must be from the baroclinic circulation of the other TC in the binary system (Fig. 1), since there was no large-scale environmental VWS in the simulations. Figure 5 shows the evolution of the upper-layer VWS (between 100 and $300 \mathrm{hPa}$ ) and the commonly used deep-layer VWS (between 200 and $850 \mathrm{hPa}$ ) averaged radially from the surface center to $600-\mathrm{km}$ radius, together with the 3-h MSLP change of the WTC in the four experiments. In stage 1, the WTC weakened with the positive 3-h MSLP change when the upper-layer VWS increased and kept a large magnitude in all experiments while the deep-layer VWS presented a decreasing trend in D480 and D600. In stage 2, the 3-h MSLP change was small and even a little bit negative, meaning that the WTC did not further weaken but experienced a quasi-steady intensity evolution. Meanwhile, the upper-layer VWS decreased first and then increased again while the deeplayer VWS continued to increase and remained large. In stage 3 , the 3-h MSLP change was mostly positive, and the upper-layer VWS increased and was larger than that in stage 2 except for D480 and D600 in which the upper-layer VWS decreased during the merging with a decrease in the deep-layer VWS. Nevertheless, in comparison with the deep-layer VWS, the evolution of the upper-layer VWS is more consistent with the 3-h MSLP change. Namely, the large or increasing upper-layer VWS corresponds to the large and positive 3-h MSLP change, and the small or decreasing upper-layer VWS corresponds to the small or even negative 3-h MSLP change. The correlation coefficients between the upper-layer VWS and the 3-h MSLP change varied from 0.6 to 0.7 at $99 \%$ confidence level in all four experiments based on the Student's $t$ test. This strongly suggests that the upper-layer VWS is a good indicator of the intensity change of the two interacting strong TCs. Note that the more detrimental effect of the upper-layer VWS on the intensity of a single mature TC has been also found in previous studies (e.g., Xu and Wang 2013; Fu et al. 2019).

The above results show a strong relationship between the upper-layer VWS and the intensity change of the simulated binary TCs. We hypothesize that the upper-layer VWS of one TC came mainly from the upper-layer anticyclonic circulation of the other TC in the binary system. Previous studies also mentioned the potential role of the VWS originated from the baroclinic structure of one TC imposed on the other TC in the binary system (WH95; Khain et al. 2000; JC15b). However, the physical processes that lead to the structure and intensity change of binary TCs have not been investigated to any extent and will be analyzed in detail in the following subsections.

\section{b. Stage 1: The early weakening of the binary TCs}

In stage 1 , one TC of the binary system was subject to the imposed vertical shear of tangential wind from the other TC, 

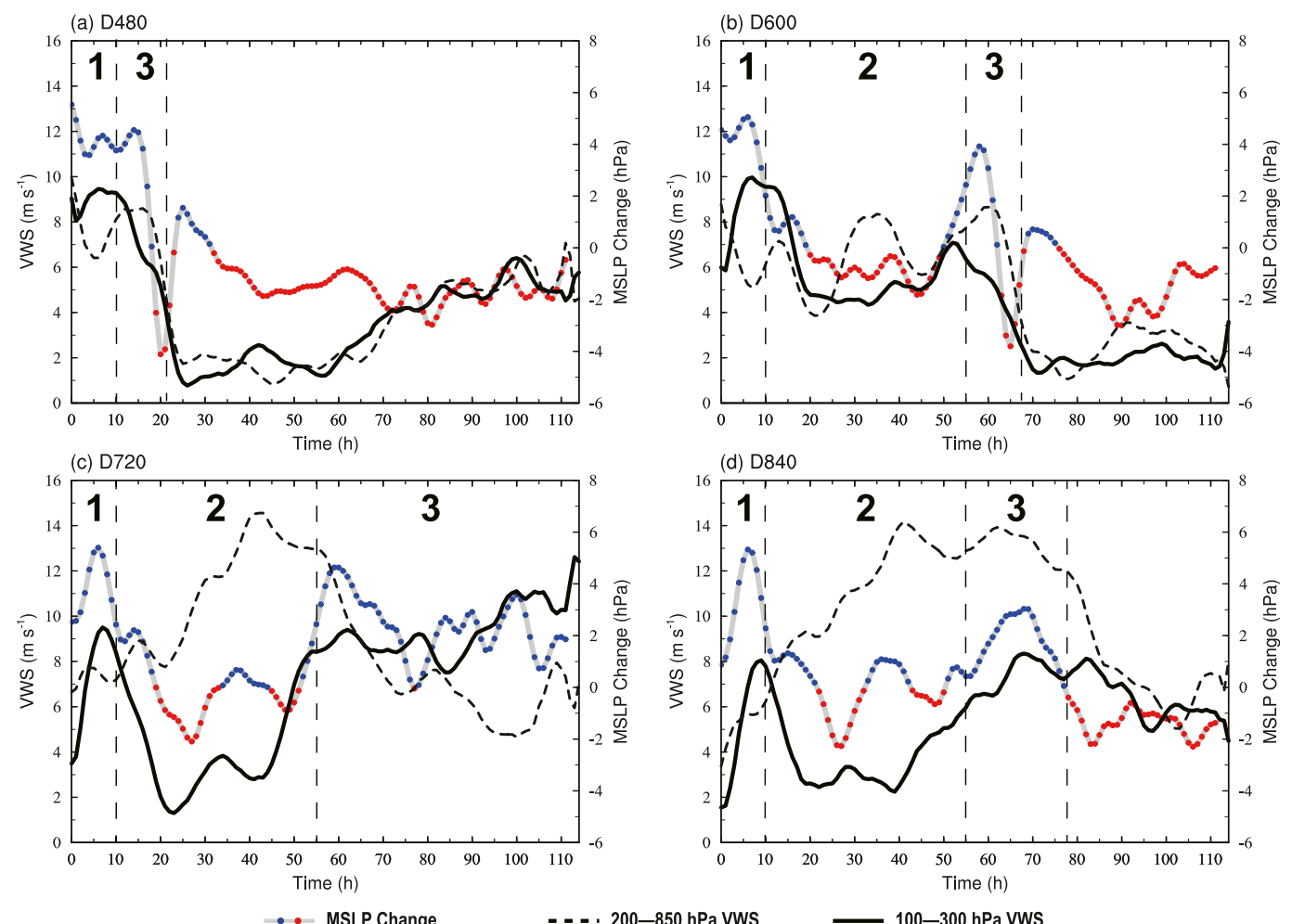

FIG. 5. Time evolution of VWS between 200 and $850 \mathrm{hPa}$ (black dashed line) and that between 100 and $300 \mathrm{hPa}$ (black solid line) over the WTC and the 3-h MSLP change (gray curves with red and blue dots) in (a) D480, (b) D600, (c) D720, and (d) D840. The red and blue dots signify intensification (negative 3-h MSLP change) and weakening (positive 3-h MSLP change), respectively.

leading to the initial ventilation of the warm core of each TC. To illustrate the impact of the ETC on the WTC, we interpolated the fields into a new coordinate system for the binary TCs with the origin following the surface center of the WTC as shown in Fig. 6. Thus, the ETC was always to the east of the WTC and the cyclonic (anticyclonic) tangential wind of the ETC across the WTC center was northerly (southerly) wind in this new coordinate system.

Figure 7 shows the potential temperature averaged from the 9- to $16-\mathrm{km}$ heights over the WTC in D720 together with the asymmetric wind vectors at the 9- and 16-km heights, respectively. Here, the asymmetric wind of the WTC was calculated as the difference between the storm-relative flow (total horizontal winds relative to the moving vortex) and the azimuthal mean wind as in Fu et al. (2019). Also plotted in Fig. 7 are the vortex center at the $16-\mathrm{km}$ height and the mean upper-layer VWS between the 16- and 9-km heights (very close to the VWS between 100 and $300 \mathrm{hPa}$ ). The vortex centers at the 9- and 16-km heights were defined as the centroid of PV for each TC in the innermost domain. Other three experiments (not shown) have similar features to D720 in stage 1.

As shown in Fig. 7, the asymmetric winds at the 16-km height ventilated the warm anomalies in the TC core, leading to the weakening of the upper-level warm core in stage 1 . The mean asymmetric winds at the $16-\mathrm{km}$ height were roughly perpendicular to the orientation between the surface centers of the binary TCs. Note that the upper-level anticyclone (ULA) of the ETC was the strongest at a height of about $16 \mathrm{~km}$ (Fig. 1a) and had southerly winds to the west of the ETC. This is consistent with the asymmetric winds across the inner core of the WTC at the $16-\mathrm{km}$ height in the early stage of the simulations, indicating the important role of the ETC's ULA on the WTC. Moreover, the ULA of the ETC induced the upper-layer VWS

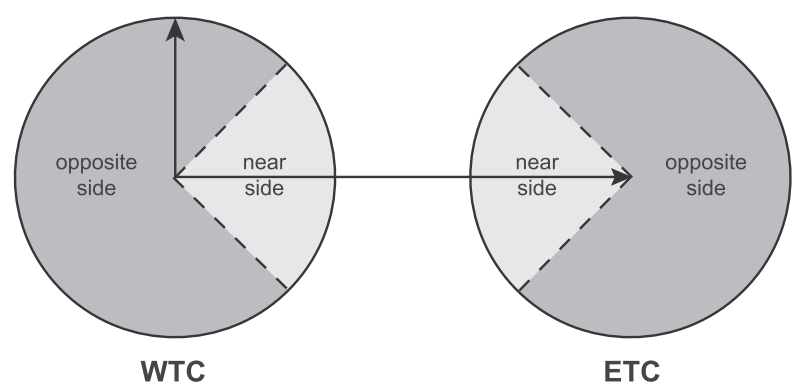

FIG. 6. Schematic diagram of the horizontal coordinate system in the analysis for binary TCs. The origin is located at the surface center of the WTC. The $x$ axis is from the WTC to the ETC. The $y$ axis is perpendicular toward the left of the direction from the WTC to the ETC. The near side (light gray zone) is the nearest quadrant of the two TCs and the opposite side (dark gray zone) is the remaining part. We define the zonal wind being along the $x$ direction and meridional wind being along the $y$ direction. 
(a)

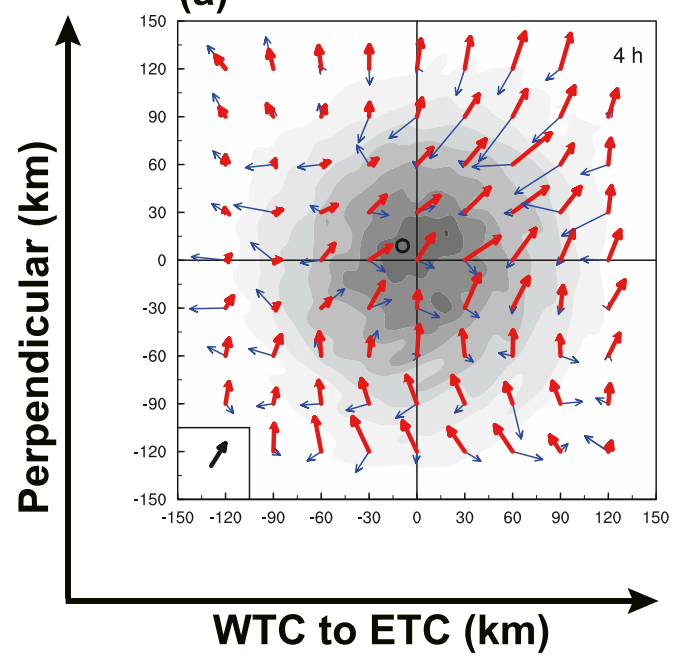

(b)

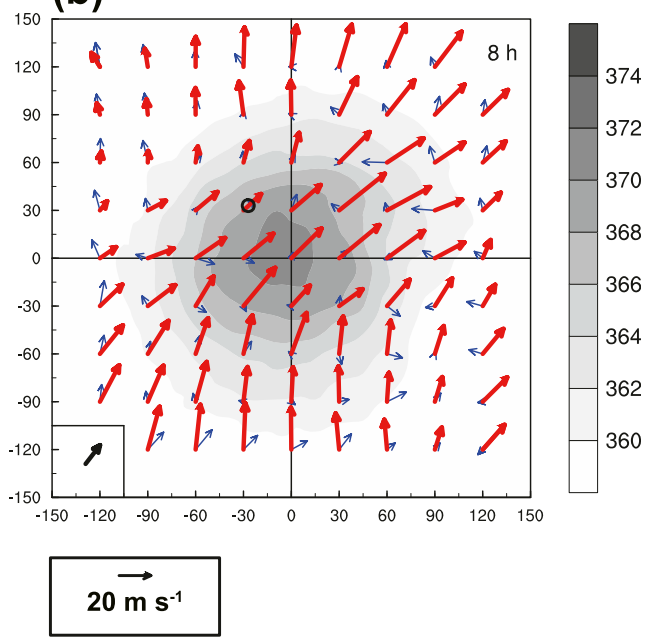

FIG. 7. Potential temperature (K, shading) of the WTC vertically averaged between the 9- and $16-\mathrm{km}$ heights at (a) 4 and (b) $8 \mathrm{~h}$ of the simulation in D720. The center of each panel is the vortex center at the 9-km height. The red and blue arrows represent the asymmetric flow at the 16- and 9-km heights, respectively. The black hollow circle denotes vortex center at the 16-km height. The black arrow in the lower-left corner of each panel is the VWS (twice longer) between the 16- and 9-km heights averaged within a radius of $600 \mathrm{~km}$ from the surface center.

over the WTC, which was also mentioned in previous studies (WH95; Khain et al. 2000; JC15b). The upper-level vortex of the WTC tilted toward the downshear-left side of the upperlayer VWS. As a result, the differential advection of the upperlevel circulation of the WTC by the VWS further enhanced the asymmetric flow crossing the warm core of the WTC. Note that the upper-layer VWS between the 16- and 9-km heights was almost equal to the mean asymmetric wind at the 16-km height because the asymmetric winds at the $9-\mathrm{km}$ height were quite weak (Figs. 4 and 7) during stage 1.

To further confirm the ventilation of the upper-level warm core by the asymmetric flow, we performed the potential temperature budget as given below following Stern and Zhang (2013):

$$
\Delta \theta=\left(\dot{\theta}_{\mathrm{ADV}}+\dot{\theta}_{\mathrm{HEAT}}+\dot{\theta}_{\mathrm{PBL}}+\dot{\theta}_{\mathrm{DIF}}\right) \Delta t
$$

where $\Delta \theta$ is the actual change in the potential temperature $(\theta)$ during the time period $\Delta t ; \dot{\theta}_{\mathrm{ADV}}$ is the total tendency due to horizontal and vertical advections of $\theta ; \dot{\theta}_{\text {HEAT }}$ is the tendency from diabatic heating; $\dot{\theta}_{\mathrm{PBL}}$ is the tendency from the boundary layer parameterization scheme; and $\dot{\theta}_{\mathrm{DIF}}$ is the tendency due to subgrid-scale horizontal diffusion. The model potential temperature tendencies are from the model output at every $6 \mathrm{~min}$, and the budget was conducted from 6 to $9 \mathrm{~h}$. The results are consistent across all simulations and thus we choose D720 as an example in the following discussion.

The patterns in 3-h actual $\theta$ change (Fig. 8a) and the results from the right-hand side (rhs) of Eq. (1) (Fig. 8b) are very similar, implying that the budget is reliable. The residual term [difference between 3-h $\theta$ change and rhs of Eq. (1), Fig. 8c] shows some errors mostly near the TC center above the $15-\mathrm{km}$ height, probably due to the interpolation and/or the tilting of the vortex at the upper levels. However, these errors do not have impact on our interpretation. Among all four terms on the rhs of Eq. (1) (Figs. 8d-f), only the total advection term $\left(\dot{\theta}_{\mathrm{ADV}}\right)$ induced significant cooling in the upper-layer eye region and the whole eyewall (Fig. 8d). The cooling in the eyewall by total advection was largely offset by diabatic heating (Fig. 8e). The boundary layer process $\left(\dot{\theta}_{\mathrm{PBL}}\right)$ and horizontal diffusion $\left(\dot{\theta}_{\mathrm{DIF}}\right)$ caused warming in the inflow layer and cooling at the top of the boundary layer (mainly contributed by $\dot{\theta}_{\mathrm{PBL}}$ term) but their tendencies were quite small compared to $\dot{\theta}_{\mathrm{ADV}}$ and $\dot{\theta}_{\mathrm{HEAT}}$ (Fig. 8f). The total advection $\left(\dot{\theta}_{\mathrm{ADV}}\right)$ can be further decomposed into the azimuthal mean radial advection $\left[\dot{\theta}_{\mathrm{RADVM}}=-\bar{u}(\partial / \partial r) \bar{\theta}\right]$, the eddy radial advection $\left[\dot{\theta}_{\mathrm{RADVE}}=-(\partial / \partial r)\left(\overline{u^{\prime} \theta^{\prime}}\right)-\left(\overline{u^{\prime} \theta^{\prime}} / r\right)\right]$ and the total vertical advection $\left(\dot{\theta}_{\mathrm{VADV}}\right)$, where $\bar{u}$ and $u^{\prime}$ are azimuthal mean and asymmetric radial wind, $\bar{\theta}$ and $\theta^{\prime}$ are azimuthal mean and asymmetric potential temperature, and $r$ is the radius from the surface TC center. The value of $\dot{\theta}_{\text {RADVM }}$ was much smaller than other two terms (Fig. $8 \mathrm{~g}$ ) because the azimuthal mean radial wind could neither pass through the TC eye region nor ventilate the warm core. Only $\dot{\theta}_{\text {RADVE }}$ contributed negatively to the tendency of the upper-level warm core (Fig. 8h) because the asymmetric flow at the 16-km height brought the cold air in and took the warm air out of the eye region (Fig. 7). $\dot{\theta}_{\text {VADV }}$ had an opposite distribution to $\dot{\theta}_{\text {RADVE }}$, but its magnitude was smaller than that of $\dot{\theta}_{\mathrm{RADVE}}$, giving rise a net negative advection tendency in the upper-level eye region and the whole eyewall. Therefore, based on the potential temperature budget, we can conclude that the eddy radial advection of potential temperature $\left(\dot{\theta}_{\mathrm{RADVE}}\right)$ due to the upper-level asymmetries (Figs. 4 and 7) is the major process that caused the weakening of the upper-level warm core and thus the intensity of binary TCs in stage 1 . The asymmetries of one TC resulted from the upper-layer VWS (Fig. 5), which in 

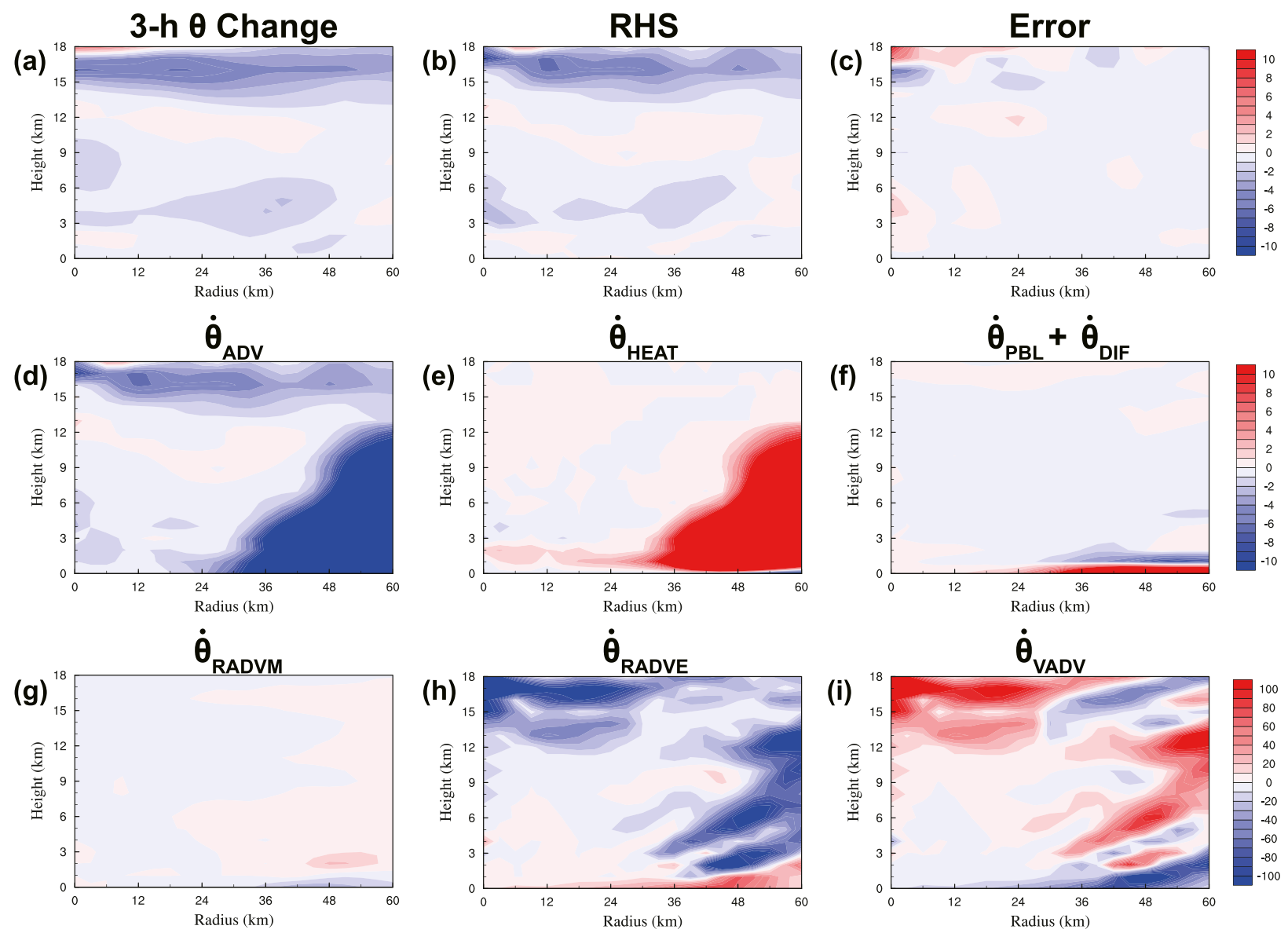

FIG. 8. Radial-height distribution of potential temperature budget terms (K, shading). (top) Azimuthal mean of (a) the actual 3-h potential temperature change, (b) the sum of the rhs of Eq. (1), and (c) budget errors. (middle) Azimuthal mean potential temperature tendencies due to (d) total advection $\left(\dot{\theta}_{\mathrm{ADV}}\right),(\mathrm{e})$ diabatic heating $\left(\dot{\theta}_{\mathrm{HEAT}}\right)$, and (f) the sum of the boundary layer process and horizontal diffusion $\left(\dot{\theta}_{\mathrm{PBL}}+\dot{\theta}_{\mathrm{DIF}}\right)$. (bottom) Azimuthal mean tendencies due to (g) the azimuthal mean radial advection $\left(\dot{\theta}_{\mathrm{RADVM}}\right)$, (h) eddy radial advection $\left(\dot{\theta}_{\mathrm{RADVE}}\right)$, and (i) total vertical advection $\left(\dot{\theta}_{\mathrm{VADV}}\right)$. All terms are budgeted for the WTC and integrated for 3-h period from 6 to $9 \mathrm{~h}$ of the simulation in D720.

turn was originated from the baroclinic circulation of the other TC in the binary system (Fig. 7).

\section{c. Stage 2: The quasi-steady intensity evolution of the binary $T C s$}

During 10 to $55 \mathrm{~h}$ of the simulations, the binary TCs in D600, D720, and D840 experienced a quasi-steady intensity evolution. We still take D720 as an example since the results are similar for D600 and D840. The upper-level warm core of the WTC changed very slowly during this period (Figs. 3c and 9). The asymmetric flow at the 9-km height strengthened during this period, which can also be found in Fig. 4c. The VWS between the 16- and 9-km heights decreased first and then increased, with the shear direction changing with time from southwesterly to northerly. As a result, the vortex centers at the 16- and 9-km heights gradually aligned and then departed again. This implies that the vortex tilting did not continue to increase in stage 2 . The relatively weak upper-layer VWS could still lead to the slow weakening of the upper-level warm core and the intensity of the TCs (Figs. 3c and 9), but its effect was largely counteracted by the faintly strengthening of the lowerlevel warm core. Potential temperature budget (not shown) indicates that the strengthening of the lower-level warm core was contributed by $\dot{\theta}_{\mathrm{VADV}}$, which was caused by the relatively weak downward motion in the eye region. This downdraft was a consequence of the reconstructed secondary circulation under the weakening of upper-level VWS. Therefore, the intensity of binary TCs showed a quasi-steady intensity evolution in stage 2 .

Changes in the upper-layer VWS over the WTC in stage 2 can be largely inferred from the evolution of asymmetric flow at the height of $9 \mathrm{~km}$ because the asymmetric flow over the WTC at the $16-\mathrm{km}$ height did not change much. At the beginning of stage 2 (Fig. 9a), the mean asymmetric wind across the center of the WTC was weaker than that at the $16-\mathrm{km}$ height and hence the upper-layer VWS was southwesterly. However, the asymmetric flow over the WTC at the 9-km height strengthened and became southerly (Figs. 9b-e), which corresponded to the ULA of the ETC. This led to a decrease in the difference of the mean winds between the 16- and 9-km 
(a)

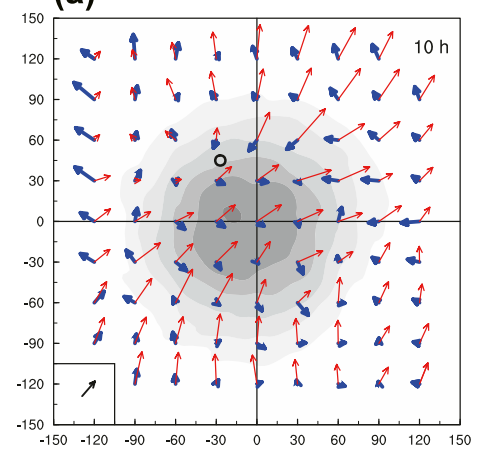

(d)

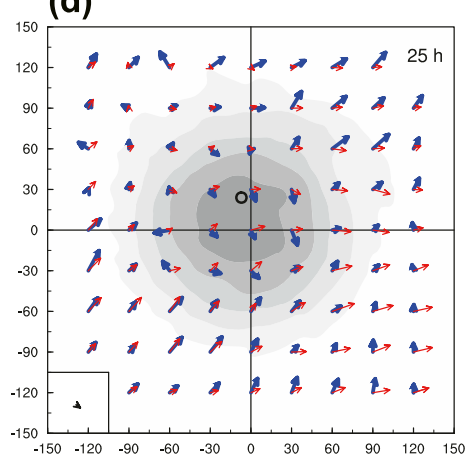

(g)

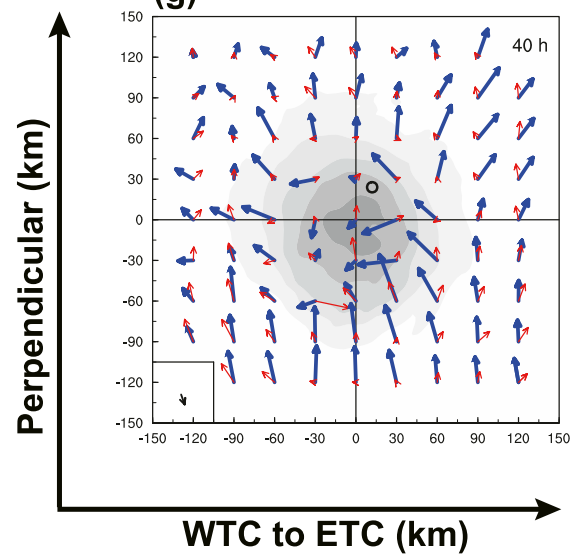

(b)

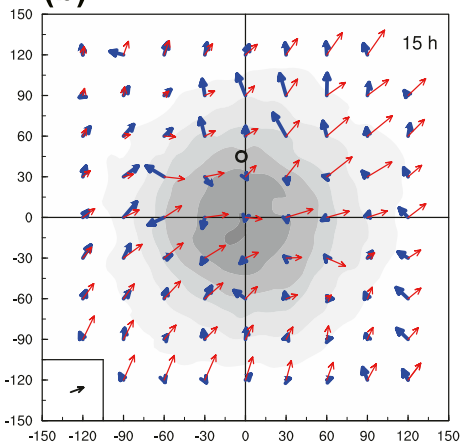

(e)

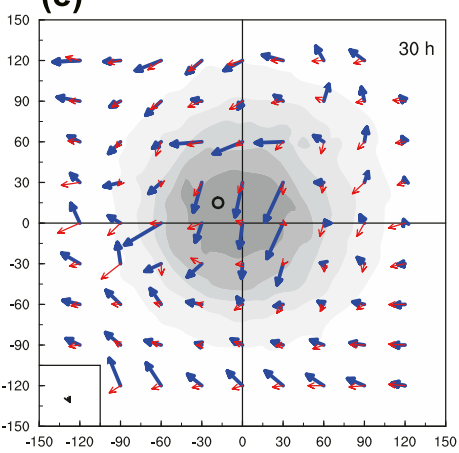

(h)

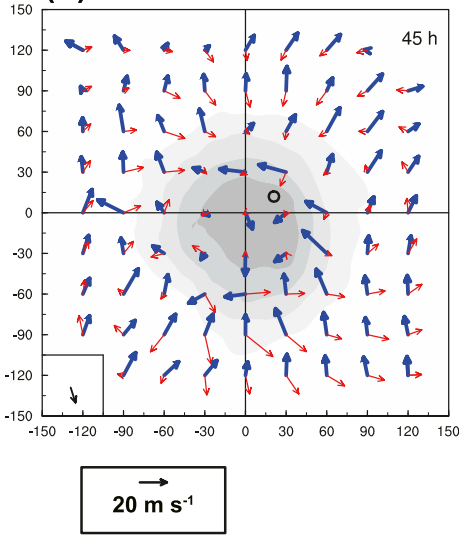

(c)

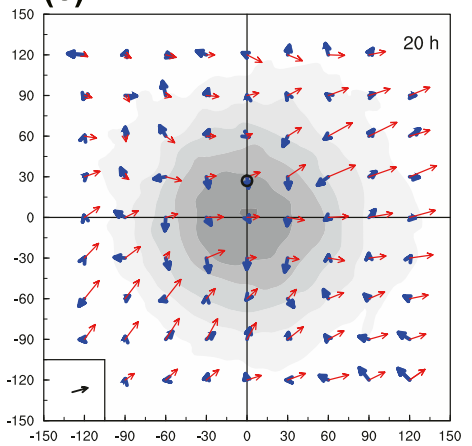

(f)

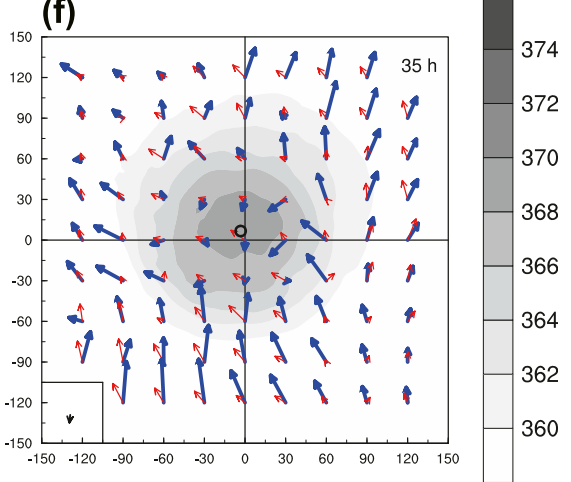

(i)

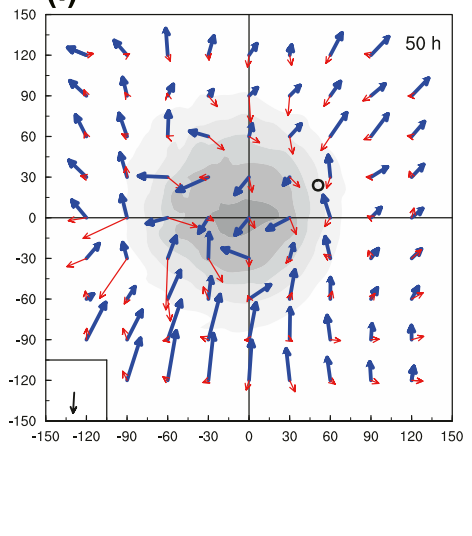

FIG. 9. As in Fig. 7, but from (a) 10 to (i) $50 \mathrm{~h}$ of the simulation at 5-h interval.

heights over the WTC, which means that the upper-layer VWS over the WTC decreased because the asymmetric flow at the $9-\mathrm{km}$ height strengthened. As the asymmetric wind at the 9-km height continued strengthening as the ULA of the ETC expanded downward (see discussion below) and became stronger than that at the $16-\mathrm{km}$ height, the upper-layer VWS over the WTC changed to northerly with the shear magnitude increasing again (Figs. 9f-i).

Since the upper-layer VWS over the WTC results from the ULA of the ETC in the binary system, it is necessary to examine how the ULA of the ETC evolved to lead to the variation of the upper-layer VWS over the WTC in stage 2. Figure 10 shows the radius-height cross sections of the azimuthal mean tangential and radial winds of the ETC on the near and opposite sides to the WTC center. The near side of the ETC was defined as the quadrant close to the WTC and the opposite side was the rest part of the ETC away from the WTC, as shown in light and dark gray zones in Fig. 6, respectively. The interaction of binary TCs should have much stronger impact on the near side, but relatively weaker impact on the opposite side of each TC. In stage 2, the opposite-side ULA of the ETC strengthened and expanded outward in D600, D720, and D840, similar to that for a single TC simulated in CTRL (not shown), but weaker because the TC in the binary system weakened due to binary interaction. The azimuthal mean anticyclonic tangential wind on the opposite side of the ETC at the 9-km height also increased but was still weaker than that at the $16-\mathrm{km}$ height, which is different from the asymmetric flow 
(a)

D600

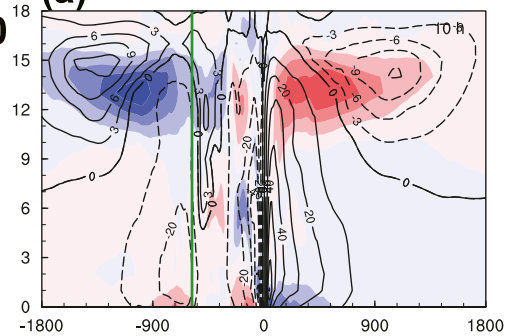

(d)

D720

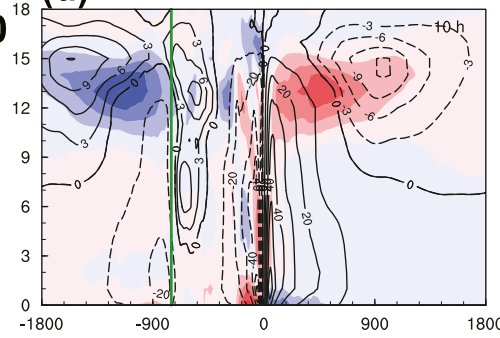

(g)

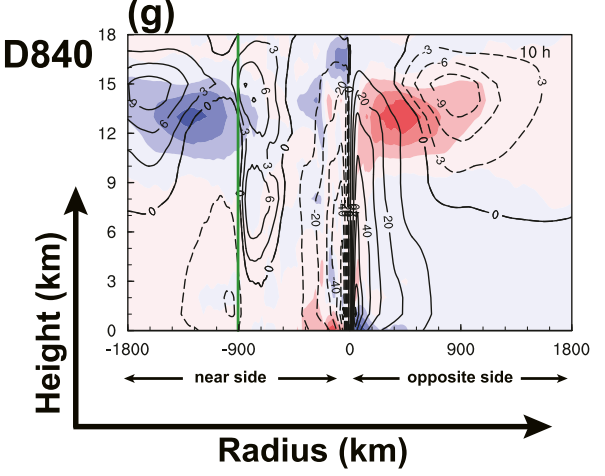

(b)

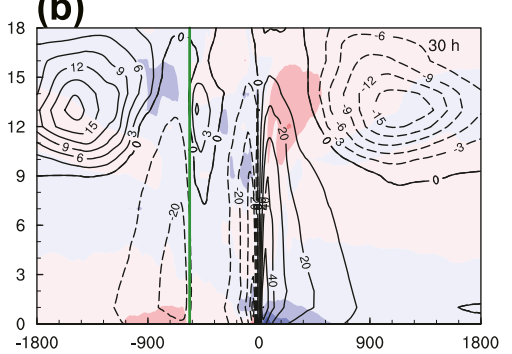

(e)
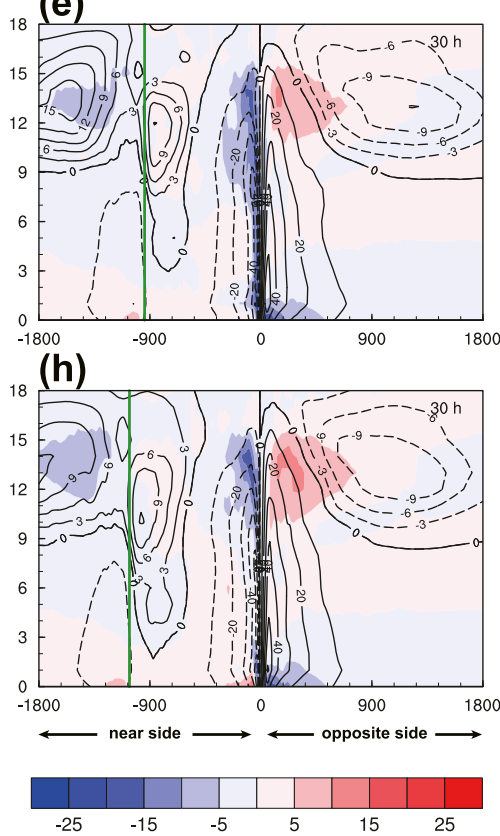

(c)

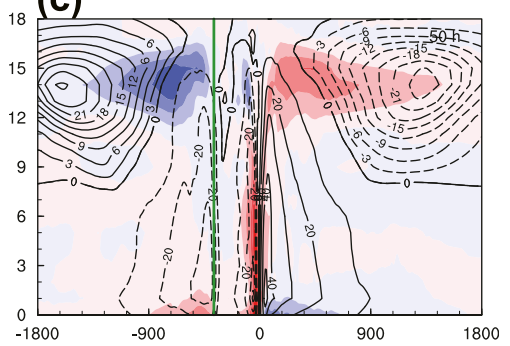

${ }_{18}(\mathbf{f})$

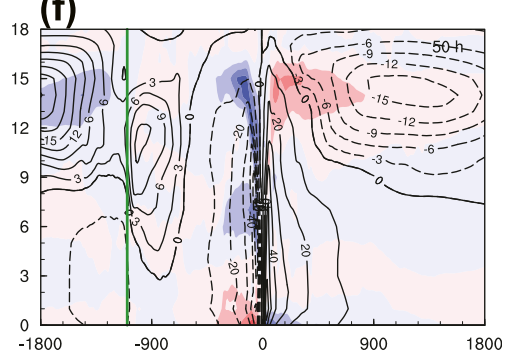

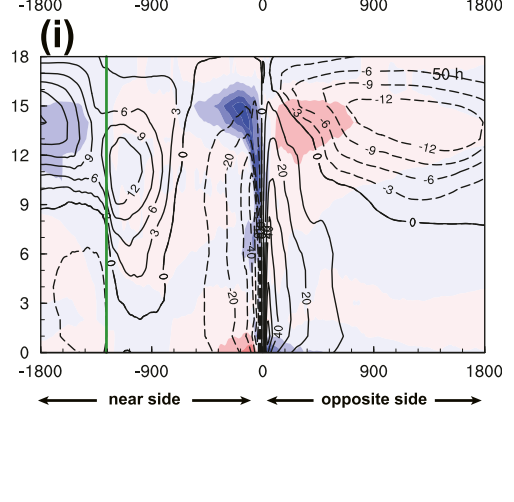

FIG. 10. As in Fig. 1a, but for the ETC from (left) 10 to (right) $50 \mathrm{~h}$ of the simulations at 20-h interval in (a)-(c) D600, (d)-(f) D720, and (g)-(i) D840. The left half of each panel is the azimuthal mean on the near side and the right half is that on the opposite side. The green line denotes the location of the WTC surface center. The result is from the outermost domain as the ULA covers a large area.

at the 9- and 16-km heights over the WTC. This implies that the tangential wind of the ETC could not fully explain changes in the upper-layer VWS over the WTC. Therefore, the binary interaction must play some role in changing the upper-layer VWS over the WTC. The azimuthal mean tangential wind on the near side of the ETC was quite different from that on the opposite side. On the near side, the ULA of the ETC expanded downward and the azimuthal mean anticyclonic tangential wind at the 9-km height became stronger over the WTC surface center in D600, D720, and D840 (Figs. 10a,d,g), leading to a decrease in the upper-layer vertical shear of the azimuthal mean tangential wind of the ETC between the 16- and 9-km heights over the WTC. As the azimuthal mean anticyclonic tangential wind of the ETC at the 9-km height continued to strengthen and became stronger than that at the 16-km height (Figs. 10e,f,h,i), the upperlayer vertical shear of the azimuthal mean tangential wind of the ETC over the WTC turned to the opposite direction and increased again in D720 and D840. This is consistent with changes in the asymmetric flows at the 9- and 16-km heights (Fig. 9) and the upper-layer VWS over the WTC (Figs. 5c,d) in D720 and D840. Different from that in D720 and D840, as the two TCs in D600 approached each other, the WTC entered the area of the upperlevel outflow of the ETC by the end of stage 2 (Fig. 10c). The outflow of the ETC at the $16-\mathrm{km}$ height was stronger than that the 9-km height over the WTC, resulting in a restrengthening of the upper-layer VWS over the WTC in D600.

Note that at the beginning of the simulations, the difference in the azimuthal mean tangential wind of the ETC between the near and opposite sides is primarily due to the superposition of winds of the two TCs, that is, the near-side ULA of the ETC was strengthened by the superposition of the cyclonic circulation on the near side of the WTC. However, this cannot explain why the ULA expanded downward. We hypothesize that the weakening of the secondary circulation in each TC, which caused downdraft anomaly in the near side of each TC and induced the downward advection of the ULA. Figure 11 shows the distributions of the vertical motion vertically averaged between the 16- and 9-km heights over the WTC in D600, D720, and D840 at 20-h interval from 10 to $50 \mathrm{~h}$ of the simulations. In stage 2, the updraft on the near side in the WTC eyewall became weaker than that on the opposite side. Downward motion anomalies occurred on the near side of the 
(a)

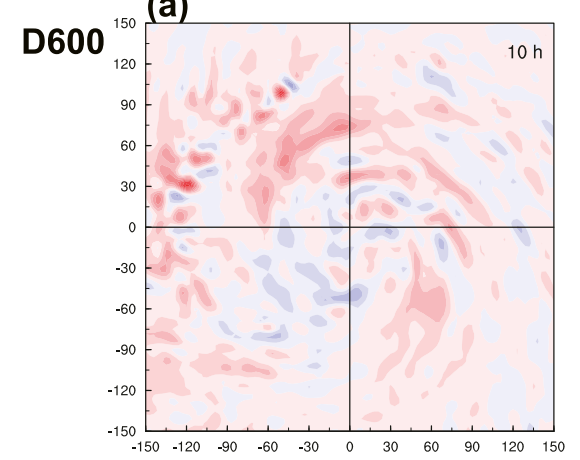

(d)

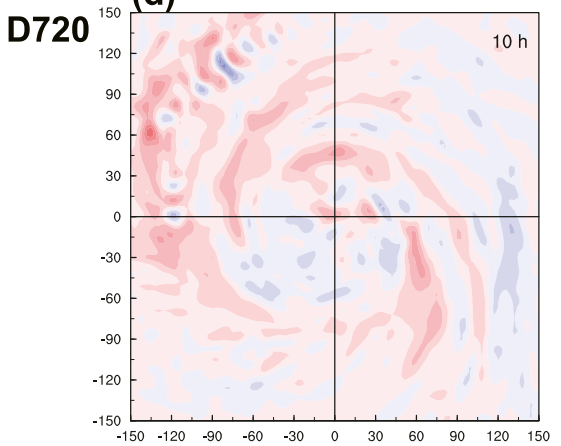

(g)

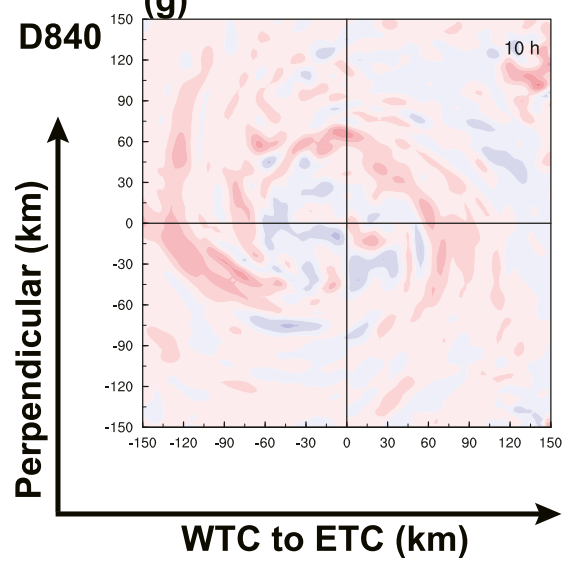

(b)

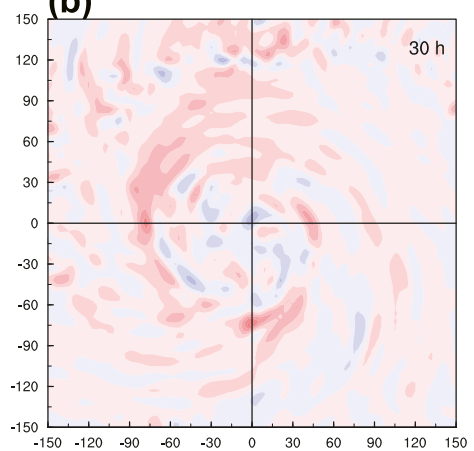

(e)

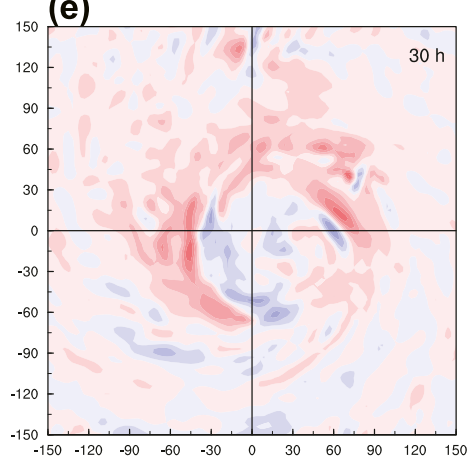

(h)

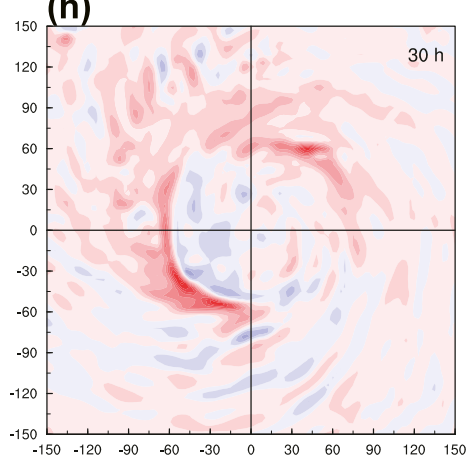

(c)

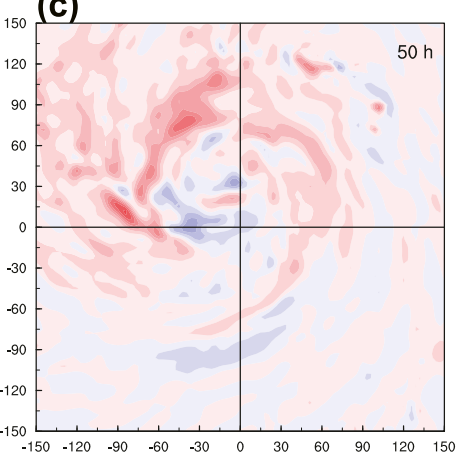

(f)

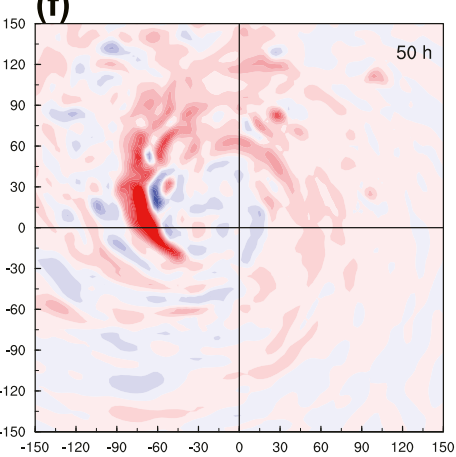

(i)

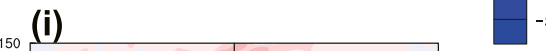

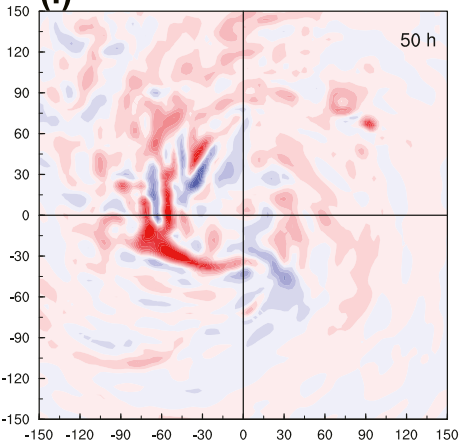

$-5$

FIG. 11. Vertical motion $\left(\mathrm{m} \mathrm{s}^{-1}\right)$ distribution relative to the surface center of the WTC vertically averaged between the 9- and 16-km heights from (left) 10 to (right) $50 \mathrm{~h}$ of the simulations at 20-h interval in (a)-(c) D600, (d)-(f) D720, and (g)-(i) D840.

WTC, especially in the southeast quadrant because of the confluence of the upper-level outflows of the WTC and the ETC. This resulted in the weakening of the secondary circulation of both TCs, and thereby the weakening of updraft on the near side of the WTC. The downward motion anomaly favored the downward penetration of the ULA (and southerly) of the ETC from the top down. This thus led to changes in the upper-layer VWS over the WTC as mentioned above.

The above process can be confirmed by the meridional wind $(V)$ budget in the binary coordinate system as defined earlier. The budget equation can be given as

$$
\Delta V=\left(\dot{V}_{\mathrm{HADV}}+\dot{V}_{\mathrm{VADV}}+\dot{V}_{\mathrm{PRES}}+\dot{V}_{\mathrm{CORI}}+\dot{V}_{\mathrm{PBL}}+\dot{V}_{\mathrm{DIF}}\right) \Delta t \text {, }
$$

where $\Delta V$ is the actual change in $V$ over a given period $\Delta t$; $\dot{V}_{\text {HADV }}$ and $\dot{V}_{\text {VADV }}$ are the tendencies due to horizontal and vertical advections, respectively; $\dot{V}_{\text {PRES }}$ and $\dot{V}_{\text {CORI }}$ are the tendencies due to pressure gradient and Coriolis forces, respectively; $\dot{V}_{\mathrm{PBL}}$ is the tendency from the boundary layer parameterization scheme; and $\dot{V}_{\text {DIF }}$ is the tendency due to subgrid-scale horizontal diffusion. All terms of the rhs of Eq. (2) are based on the model output at every $6 \mathrm{~min}$ as in the potential temperature budget. Figure 12 shows the $V$ budget of the WTC at the 9- and 16-km heights in D600, D720, and D840 in stage $2(42-48 \mathrm{~h})$. The difference between the model $V$ change and the sum on the rhs of Eq. (2) integrated from 42- to 48-h simulations was quite small. At the 9-km height, all three experiments show the increasing in $V$ (southerly or anticyclonic 


\section{$9 \mathrm{~km}$}
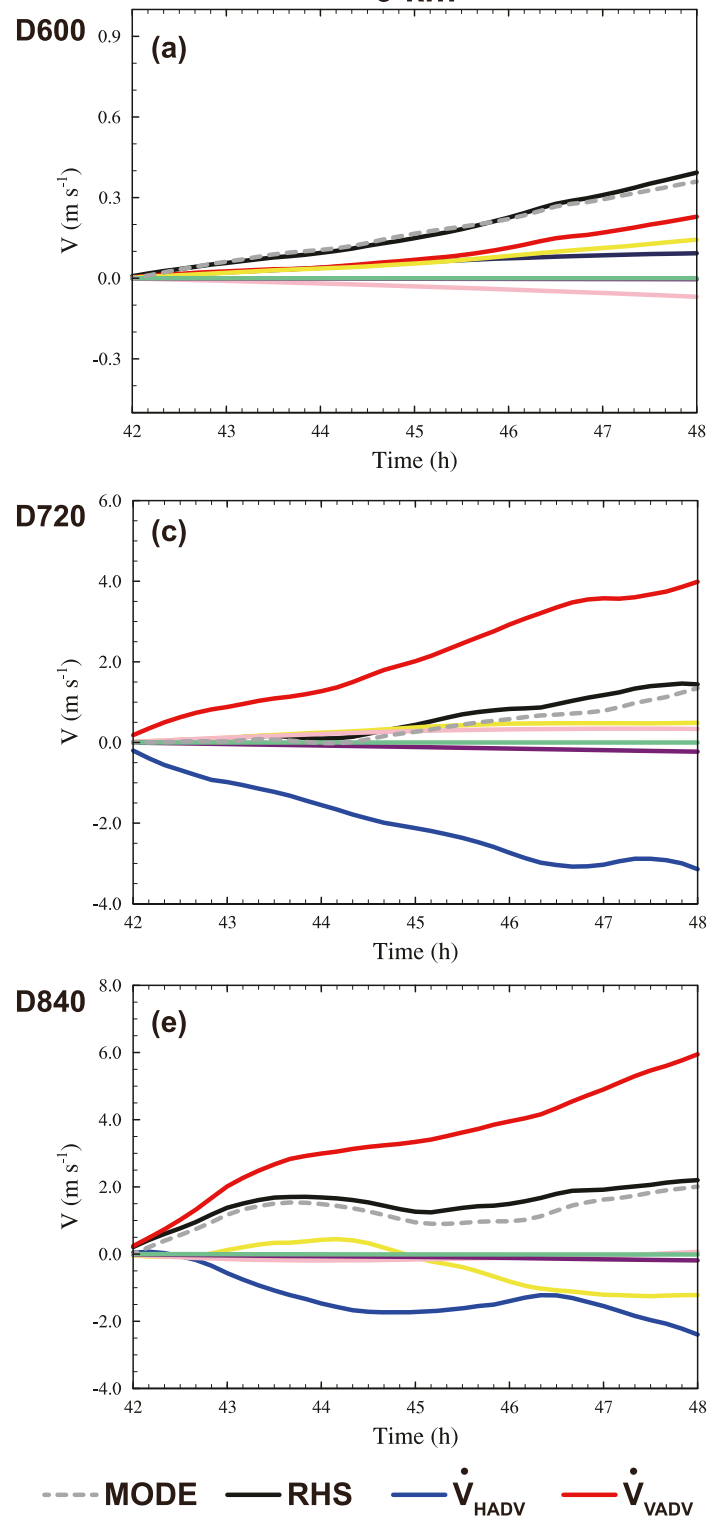
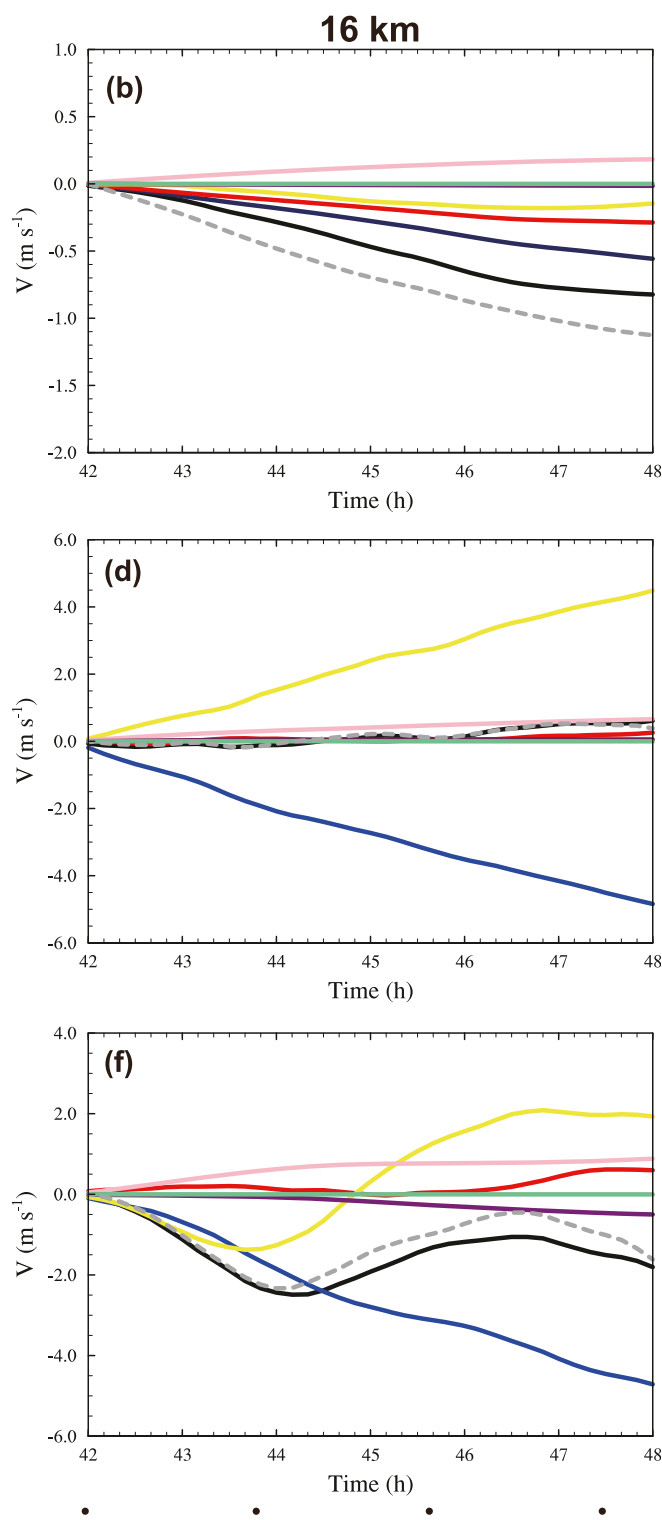

FIG. 12. Time evolutions of the actual inner-core mean meridional wind $(V)$ change (MODE, dashed gray line) relative to $42 \mathrm{~h}$ of the simulation of the WTC, the sum of the azimuthal mean rhs of Eq. (2) (black solid line), $\dot{V}_{\text {HADV }}$ (blue solid line), $\dot{V}_{\text {VADV }}$ (red solid line), $\dot{V}_{\text {PRES }}$ (yellow solid line), $\dot{V}_{\text {CORI }}$ (pink solid line), $\dot{V}_{\text {PBL }}$ (purple solid line), and $\dot{V}_{\text {DIF }}$ (green solid line) in Eq. (2) in (a),(b) D600, (c),(d) D720, and (e),(f) D840 at the (left) 9- and (right) 16-km heights. See text for details.

tangential wind relative to the ETC). $\dot{V}_{\text {VADV }}$ was positive during the budget period and was the primary cause for the increase in $V$ (viz., southerly strengthened). Other terms on the rhs of Eq. (2) contributed little or even negative tendency to the southerly wind. At the $16-\mathrm{km}$ height, $V$ changed little in D720 (Fig. 12d) and decreased in both D600 and D840 (Figs. 12b,f). The tendencies contributed by $\dot{V}_{\text {PRES }}$ and $\dot{V}_{\text {HADV }}$ were largely offset in both D720 and D840. Therefore, the momentum budget of meridional wind demonstrates that the enhancement of asymmetric southerly at the 9-km height over the WTC mainly resulted from the downward advection of the near-side ULA of the ETC in stage 2. Namely, the binary interaction weakened the near-side vertical secondary circulation of each TC in stage 2 , causing the downward expansion of the near-side ULA. As a result, the upper-layer VWS over each TC weakened first and then restrengthened, leading to marginally changes in the upperlevel warm core and the intensity of the binary TCs.

\section{d. Stage 3a: The second weakening of the nonmerging binary $T C s$}

The TCs in the two nonmerging binary experiments (D720 and D840) experienced the second weakening (stage 3). This stage can 
3-h $\theta$ Change
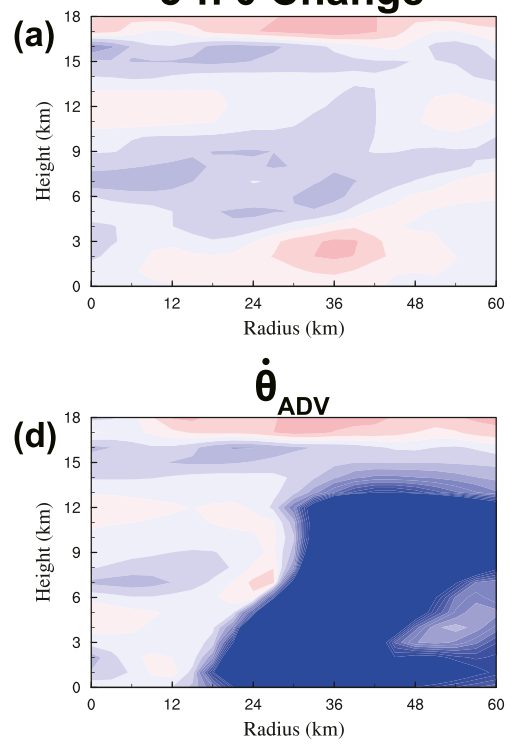

$\dot{\theta}_{\text {RADVM }}$

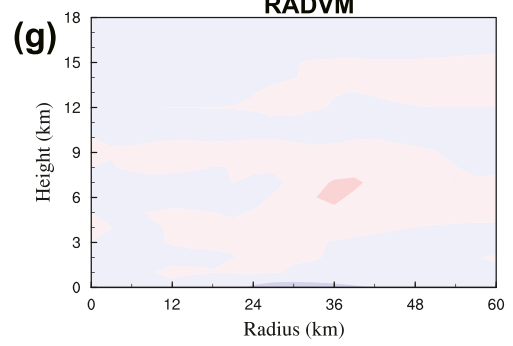

RHS

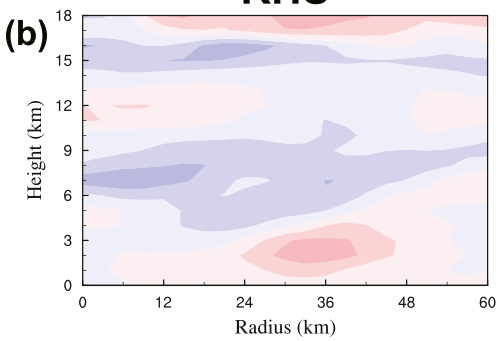

$\dot{\theta}_{\text {HEAT }}$

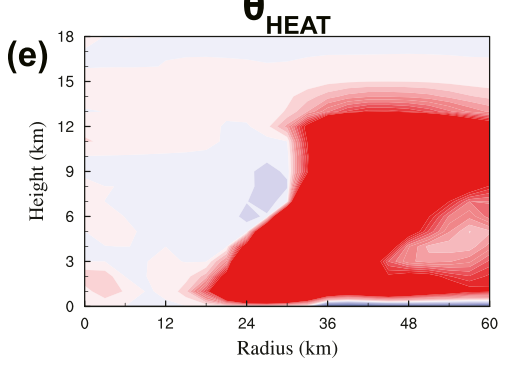

$\dot{\theta}_{\text {RADVE }}$

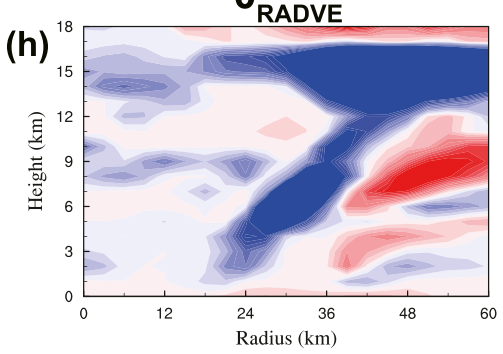

Error
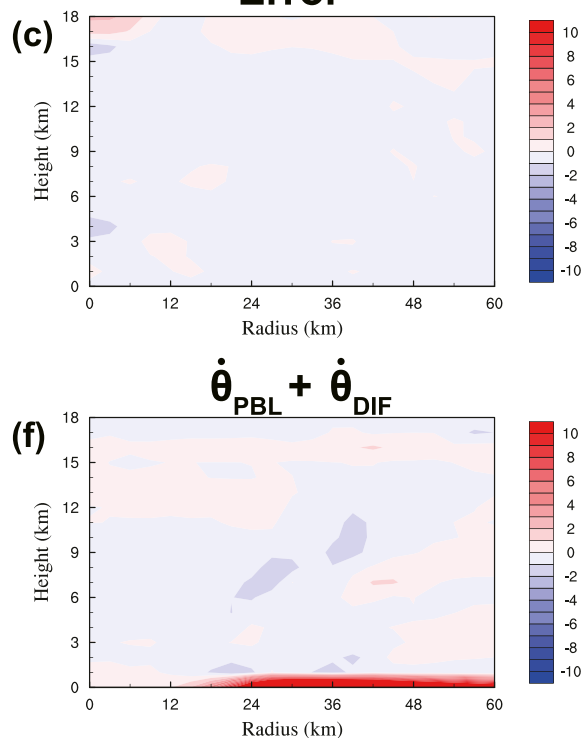

$\dot{\theta}_{\text {VADV }}$

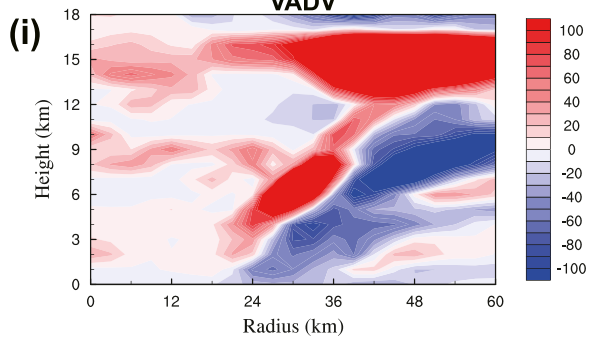

FIG. 13. As in Fig. 8, but all tendencies are integrated from 66 to $69 \mathrm{~h}$ of the simulation in D720.

be considered as a continuation of stage 2 because the upper-layer VWS continued strengthening and remained large by the end of stage 2 (Figs. 5c,d). The binary TCs in both D720 and D840 experienced a weakening in stage 3 as in stage 1 . The large upperlayer VWS led to the strengthening of the RKE above the 9-km height (Figs. 4c,d). The potential temperature budget shown in Fig. 13 indicates that $\dot{\theta}_{\mathrm{RADVE}}$ weakened the warm core at the 15and $9-\mathrm{km}$ heights of the WTC. Compared with stage 1 , the weakening of the warm core at the $9-\mathrm{km}$ height caused by $\dot{\theta}_{\text {RADVE }}$ in stage 3 further confirms that the binary interaction resulted in downward expansion of the near-side ULA as discussed for stage 2 in section $3 \mathrm{c}$.

After stage 3 in D840, the upper-layer VWS decreased and the binary TCs deepened (Fig. 5d) until the end of the simulation when the separation distance between the two TCs increased over $1600 \mathrm{~km}$ (Fig. 2h). We also examined additional experiments with the same initial vortices but different initial separation distances and found that the overall behavior of the binary TCs are quite similar (not shown) with the weakening terminated once their separation distance exceeding about $1600 \mathrm{~km}$. This seems to be a critical separation distance in our experiments and is consistent with some relevant statistical analyses of binary-TCs interaction based on observations (Brand 1970; Dong and Neumann 1983; Wu et al. 2011; Jang and Chun 2015a; Ren et al. 2020). Since the
ULA of one TC affects the intensity of the other TC in the binary system, we further introduce the local Rossby radius of deformation $L_{R}(=N H / I$, where $N$ is the Brunt-Väisälä frequency; $H$ is the scale height; and $I$ is the inertial stability) of the azimuthal mean ULA for understanding the dynamics. $N H$ is $60 \mathrm{~m} \mathrm{~s}^{-1}$ the same as that in Bell et al. (2012), and $I[=\sqrt{(f+\zeta)(f+2 \bar{v} / r)}$, where $\zeta$ is the relative vorticity and $\bar{v}$ is the azimuthal mean tangential wind] is calculated from the model output. We did not use the near-side azimuthal mean ULA because once the two TCs were far enough away from each other, the difference of the azimuthal mean ULA on the near and opposite sides was negligible. We can estimate $L_{R}$ to be $1500-1700 \mathrm{~km}$, comparable to $1600 \mathrm{~km}$ in our experiments. Note that $L_{R}$ is much smaller in the middlelower troposphere than in the outflow layer because of the much larger inertial stability. Therefore, it is expected that once the separation distance between the two TCs becomes larger than the horizontal-scale $L_{R}$ determined by the ULA, the binary interaction and the associated intensity change would become very weak and can be ignored.

\section{e. Stage 3b: The weakening of the binary TCs during merging}

From 10 to $22 \mathrm{~h}$ of the simulation in D480 and from 55 to $67 \mathrm{~h}$ of the simulation in D600, the binary TCs experienced a 
(a)

(c)

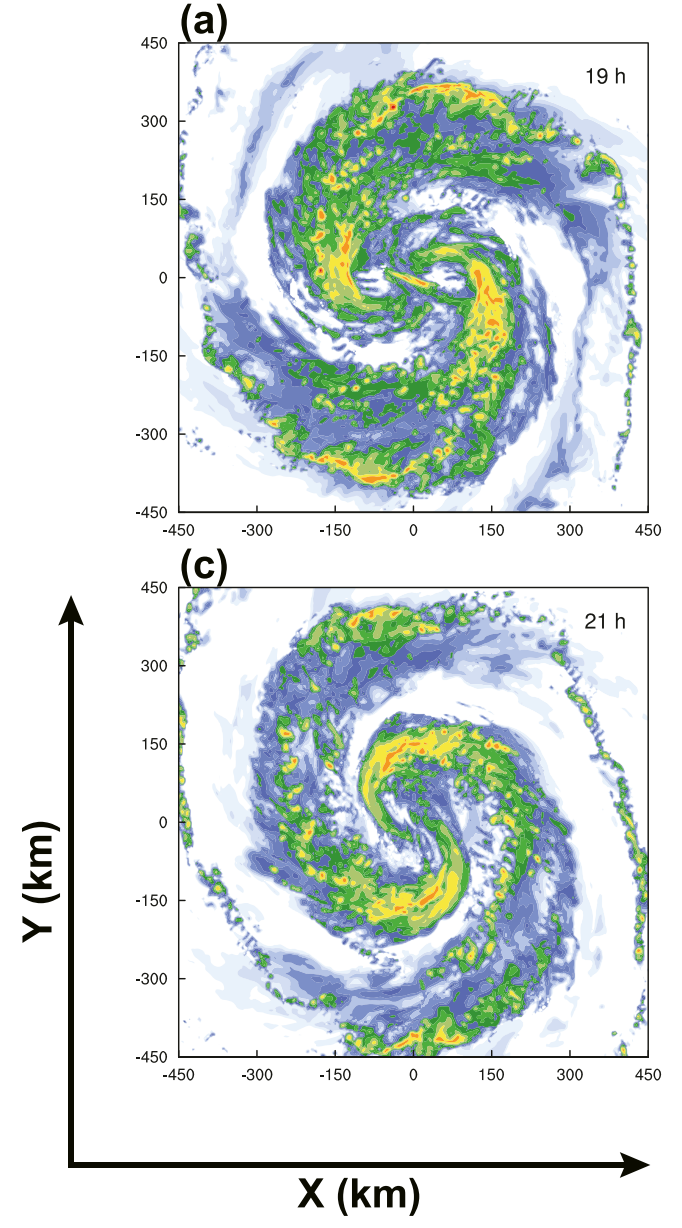

${ }_{450}($ b)

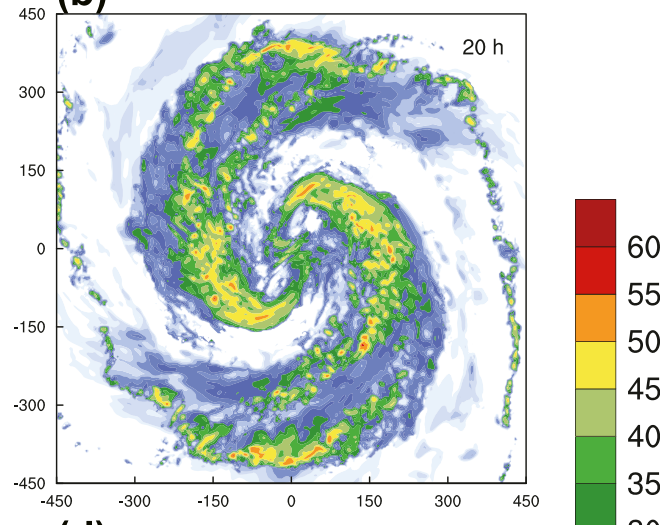

(d)

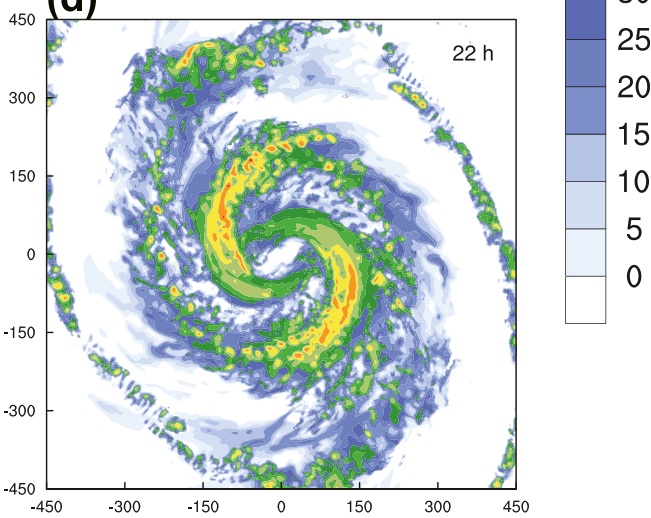

FIG. 14. Horizontal distributions of simulated maximum radar reflectivity (dBZ) every hour from (a) 19 to (d) $22 \mathrm{~h}$ in D480.

weakening period prior to their merger. During this stage, the two TCs presented increasingly asymmetric structure as they approached each other with their separation distance decreasing rapidly, consistent with the weakening of the two TCs. Since the structure and intensity evolutions are similar in D480 and D600, we take D480 as an example to describe the merging process. Figure 14 shows the simulated maximum radar reflectivity in D480 prior to merging. The binary TCs showed an " 8 "-shaped rainband structure. The eyewall on the near side of each TC was weaker than that on the opposite side and gradually weakened but suddenly disappeared before merging. The partial eyewalls of the two TCs finally formed a new closed eyewall of the merged TC system. Figure 15 shows the PV distributions in D480 at four different heights. In the boundary layer (at the 1-km height) in D480 (Fig. 15a), the two vortices presented weak deformation at $18 \mathrm{~h}$ of the simulation but then accelerated the rotation with more and more deformation and filamentation until they merged (Figs. 15b,c). The similar deformation and filamentation also occurred in the lower- (at the 2-km height, Figs. 15d-f) and middle-troposphere (at the 5-km height, Figs. $15 \mathrm{~g}-\mathrm{i}$ ), but both were weaker and the merging of the two vortices lagged that in the boundary layer. Going to higher levels, the PV structure (Figs. 15j-1) was more asymmetric as the circulation in the upper troposphere (at the 9-km height) was much weaker. The two vortices at the upper level rotated more slowly than that at the lower and middle levels, implying a large vertical tilt of the TC vortices. Different from those in the middle-lower troposphere, the PV field at the 9-km height shows no merging but strong deformation and filamentation, leading to banded structure as the low-level vortices merged. The results thus demonstrate a bottom-up merging process of the binary TCs in the simulations, which is consistent with the findings in WH95.

Figure 16 shows the vertical cross sections of the total wind speed through the surface vortex centers of the two TCs in D480. The eyewalls as inferred by large wind speed on the near side of the binary TCs weakened from the top down partly due to the vertical tilt of the TC vortices. Meanwhile, both TC vortices became more asymmetric. This highly asymmetric TC structure resulted primarily from the strong shearing deformation of the TC vortices, a process well studied with both barotropic and baroclinic models in the literature (Holland and Dietachmayer 1993; WH95; Falkovich et al. 1995; Khain et al. 2000; Prieto et al. 2003; Shin et al. 2006). After the two TCs 
(a)

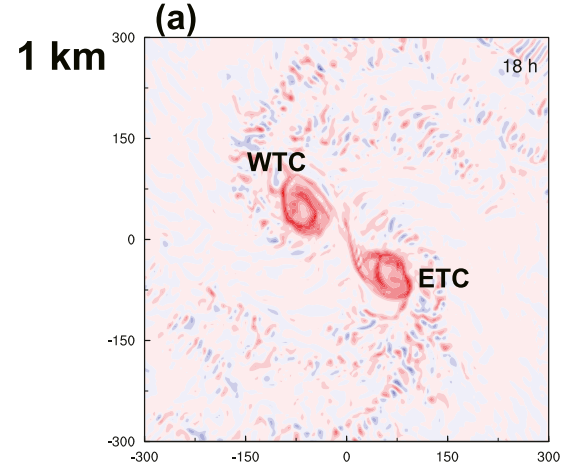

(d)

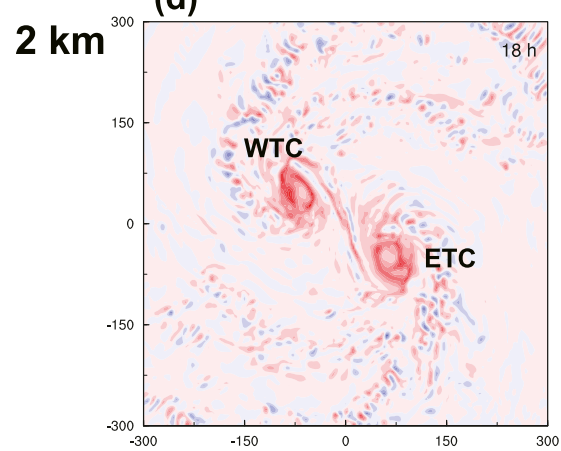

(g)
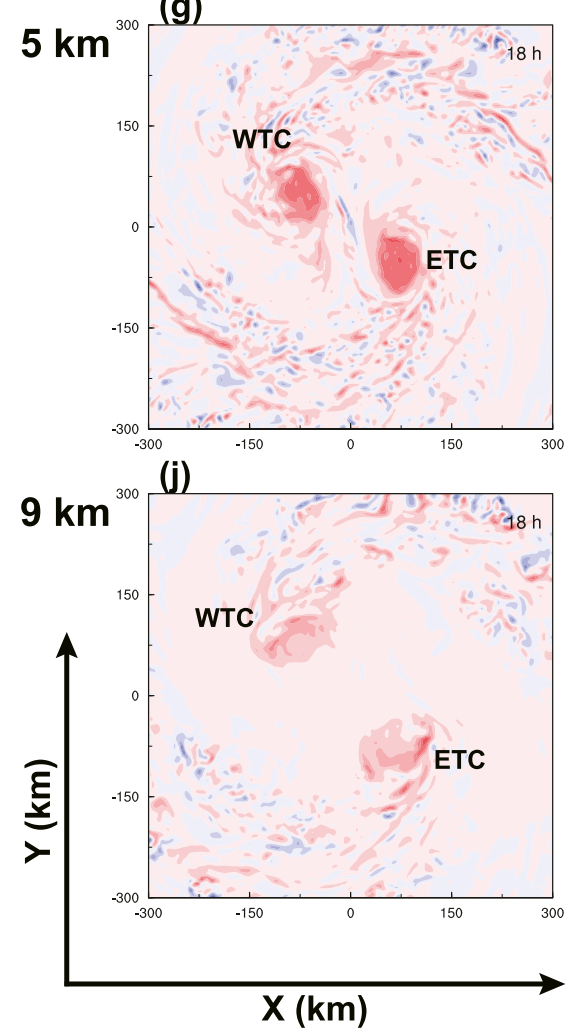

(b)

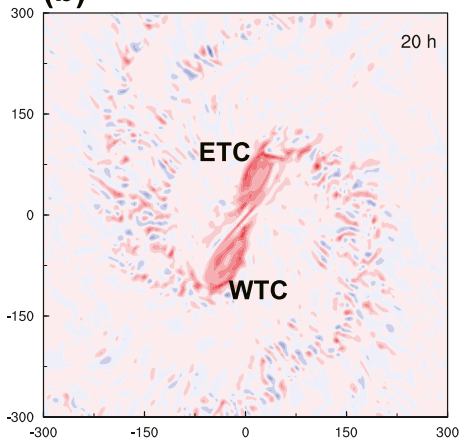

(e)
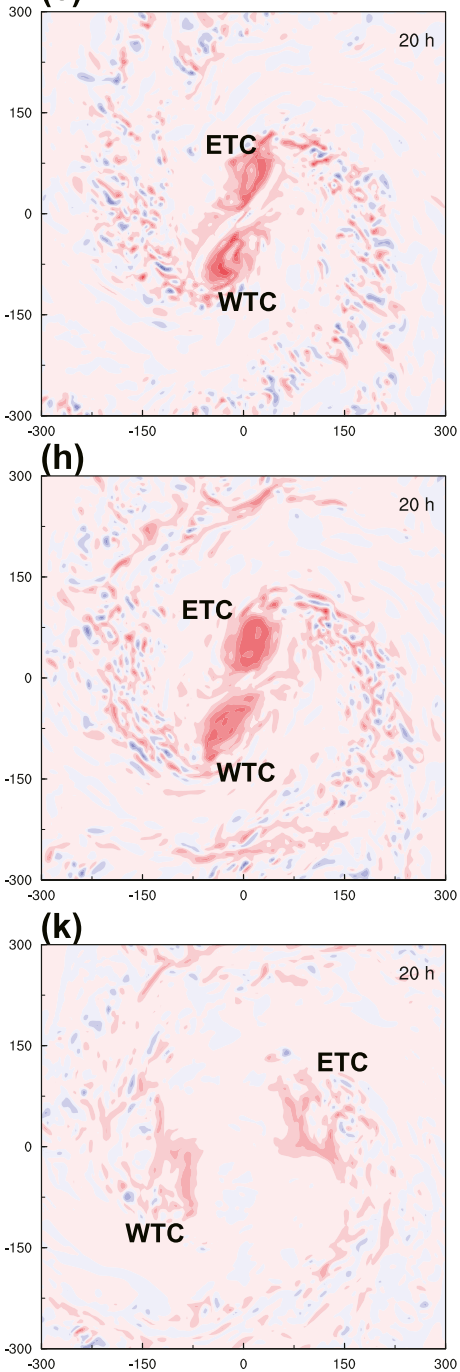

${ }_{300}($ c)

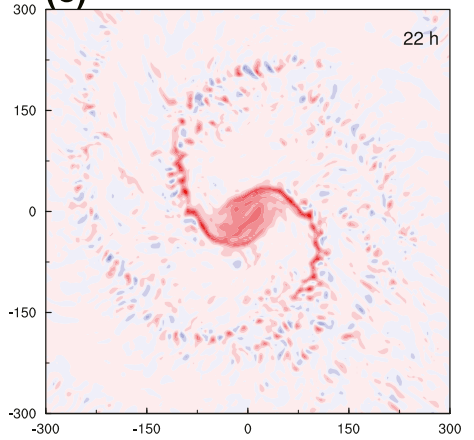

${ }_{300}(\mathrm{f})$
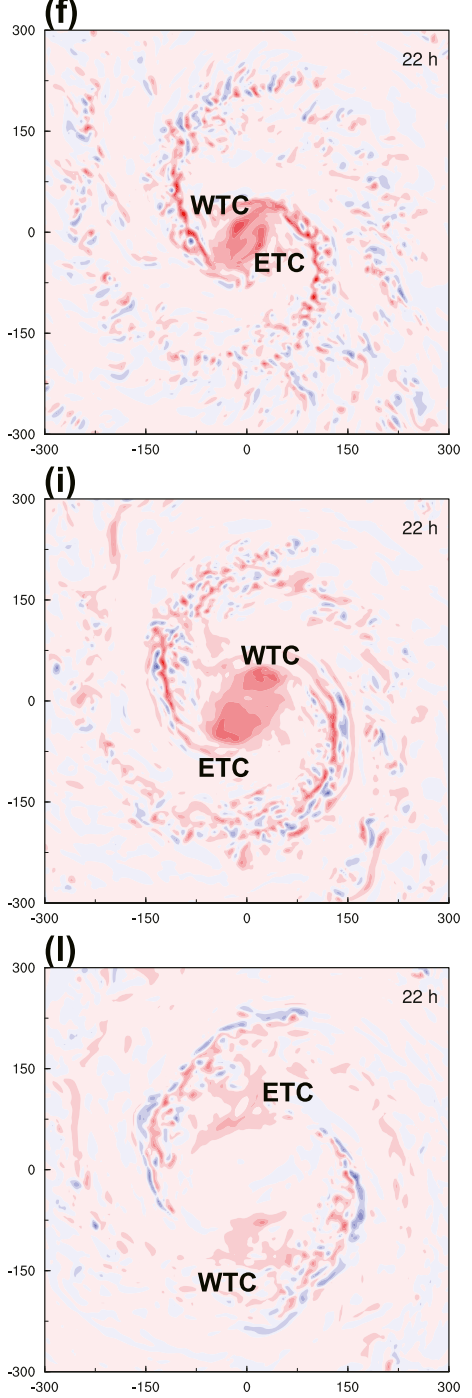

FIG. 15. Horizontal distributions of PV (PVU) at the (a)-(c) 1-, (d)-(f) 2-, (g)-(i) 5-, and (j)-(l) 9-km heights in D480 at (left) 18, (center) 20 , and (right) $22 \mathrm{~h}$ of the simulation. 
(a)

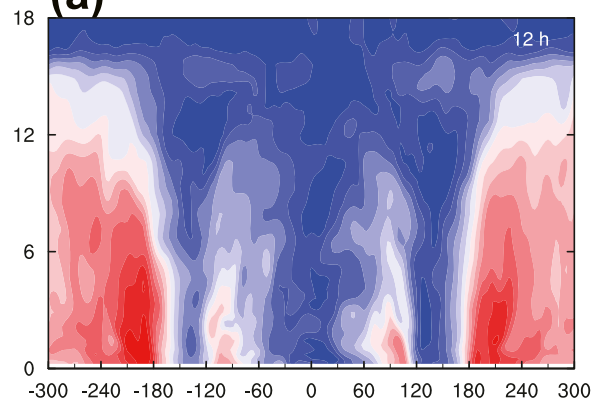

(c)

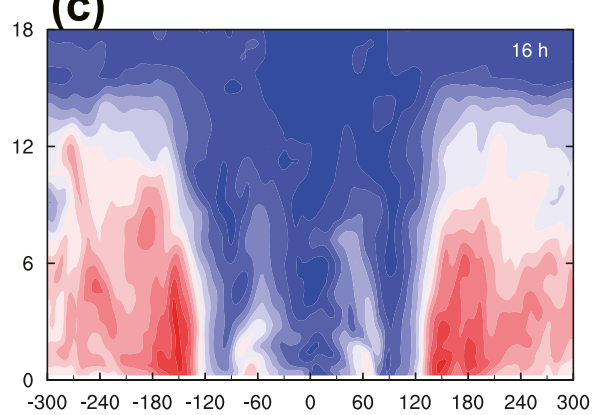

(e)

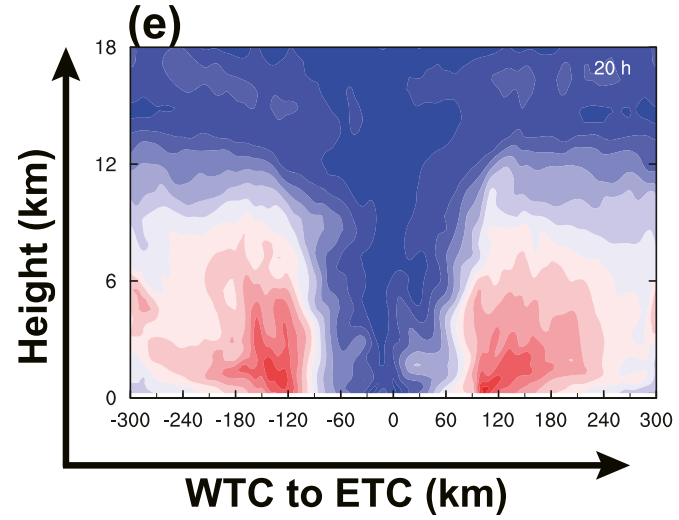

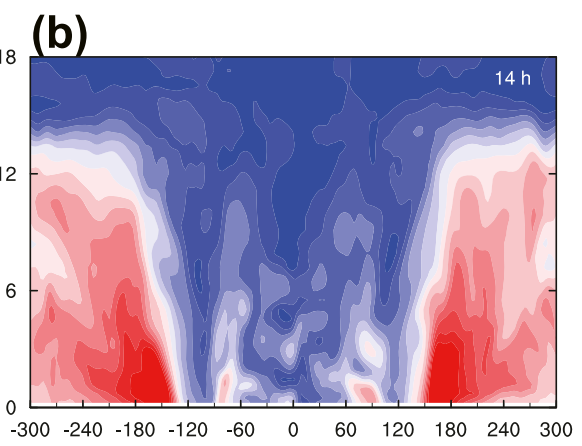
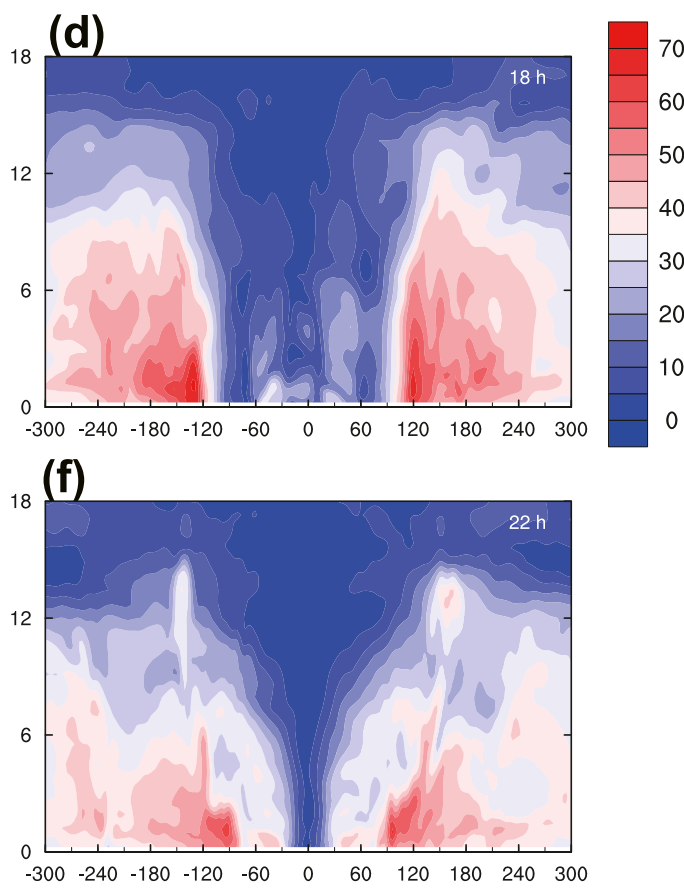

FIG. 16. Vertical cross sections of total wind speed $\left(\mathrm{m} \mathrm{s}^{-1}\right.$ ) along the $x$ axis from (left) the WTC to (right) the ETC from (a) 12 to (f) $22 \mathrm{~h}$ of the simulation at 2-h interval in D480.

merged, the new eye was larger than the eye of either TC prior to merging. The merger occurred very rapidly and the new TC became more and more axisymmetric and also experienced intensification after merger. These are consistent with those documented in previous studies (WH95; Khain et al. 2000) and can also be seen from Figs. $2 \mathrm{a}-\mathrm{d}$. Therefore, the weakening and structure change of the binary TCs prior to merging, including the highly asymmetric rainbands, the near-side eyewall weakening and breakdown from the top down, and the large tilt, were due to the combined effect of large VWS and strong horizontal shearing deformation of the drastic binary interaction when the two TCs approached rapidly toward merging.

\section{Conclusions and discussion}

In this study, the intensity change of binary TCs has been investigated using a three-dimensional numerical model with a finest grid spacing of $3 \mathrm{~km}$ under idealized conditions on an $f$ plane with a constant sea surface temperature and in a quiescent environment. To allow more realistic binary interaction, we first simulated a single TC for $96 \mathrm{~h}$ until it reached its mature stage with the fully developed secondary circulation and used its axisymmetric vortex as the initial TCs in the binary experiments. Four experiments were performed with the initial separation distances between the two identical TCs being 480 (D480), 600 (D600), 720 (D720), and $840 \mathrm{~km}$ (D840), respectively. Results show that the two TCs approached each other and merged only in D480 and D600. In D720, the two TCs rotated cyclonically but with their separation distance increasing with time. In D840, the two TCs rotated cyclonically in the early stage but then anticyclonically and moved away (escaped) from each other. These three interaction modes are very similar to the conceptual model of binary TC interaction proposed by LH93. 
The intensity of the binary TCs experienced three stages and the intensity change was well correlated with the VWS in the upper troposphere between 100 and $300 \mathrm{hPa}$ over the inner core of each TC. The first stage was the weakening of the binary TCs during the first $10 \mathrm{~h}$ of the simulations. In this stage, the vertical shear of the tangential wind from one TC imposed a large upper-layer VWS on the other TC, leading to the ventilation and weakening of the warm core through both horizontal advection and eddy flux of the potential temperature. Such a mutual interaction led to the weakening of the two TCs. The second stage was the quasi-steady intensity evolution in the three binary experiments (D600, D720, and D840) from 10 to $55 \mathrm{~h}$, when the two TCs did not yet or never merge. During this stage, the upper-layer VWS decreased first due to the enhanced asymmetric flow at the 9-km height. The increasing asymmetric flow resulted mainly from the outward and downward expansion of the ULA due to the superposition of the two TCs' circulations and the binary interaction. As a result, the vertical tilt of the TC vortex largely decreased, the warm core remained its strength, and the TCs experienced little intensity change. In the merging cases of D480 and D600, the two TCs continued weakening until the two TCs merged. The weakening was mainly due to both the VWS and shearing deformation of the cyclonic circulation as previously studied. After merging in D480 and D600, the new TC system experienced intensification due to the continuous axisymmetrization. The nonmerging binary TCs in D720 and D840 weakened again after the quasi-steady intensity evolution as the asymmetric flow at the 9-km height induced by the ULA of the opposite TC continued increasing. This led to the increase in the upper-layer VWS over each TC and thus the weakening of the warm core and the TCs. The intensity of the binary TCs in D840 did not decrease further after $78 \mathrm{~h}$ of the simulation because the interaction of the binary TCs became very weak after their separation distance increased to $1600 \mathrm{~km}$, which is comparable and even larger than the local Rossby radius of deformation $\left(L_{R}\right)$ of the upper-level anticyclonic circulation of the TCs. Therefore, $L_{R}$ determines the horizontal scale of the ULA and can be used as a measure to determine whether the two TCs could experience direct binary interaction.

Note that the intensity change of binary TCs is investigated under idealized conditions in this study. It is unclear whether the importance of upper-layer VWS in the binary interaction is a model result or also true in real binary TCs. The initial vortex in our experiments was as intense as $895 \mathrm{hPa}$ in its MSLP (about $70 \mathrm{~m} \mathrm{~s}^{-1}$ in the maximum near-surface wind speed), which seems to be "unrealistically strong" in the real atmosphere. We used such a strong TC because it is in its mature stage of a simulated TC with well-developed primary and secondary circulations. This can ensure a strong binary interaction and overcome the initial model spinup of a weak TC and also the intensity change of the binary TCs can be attributed to the binary interaction because the single TC would experience a quasi-steady intensity evolution. Note that we also conducted a series experiments of relatively weak binary TCs (initial maximum near-surface wind speed of $35 \mathrm{~m} \mathrm{~s}^{-1}$, not shown); the two TCs showed similar intensity evolutions as those shown in this study. In addition, the detailed merging processes have not been analyzed in this study as they seem to be similar to what revealed in previous studies (e.g., WH95; Khain et al. 2000; JC15b). Previous studies (Yang et al. 2008; Jang and Chun 2013; Liu and Tan 2016) also suggested that the structure and intensity changes of binary TCs could be sensitive to the initial TC structure and intensity in real atmosphere. Therefore, how sensitive the binary-TCs interaction is to the structure of the TCs and the relative intensity and size of the two TC vortices needs to be addressed in future studies. In addition, the binary interaction is often affected by the environmental flow and latitudinal dependence of the Coriolis parameter (e.g., WH95; Khain et al. 2000; JC15b). In those cases, the initial orientation and relative intensity of the two TCs may become also important (WH95). In addition, it is also worth noting that the role of the $L_{R}$ of the ULA in distinguishing the direct binary interactions is found in fully physics moist simulations, in case of binary interaction of dry TC-like vortices the ULA may be very weak or even not exist at all. It is unclear what scaling parameters can be used to distinguish the direct interaction for dry vortices. These will be examined in our future studies.

Acknowledgments. The authors are grateful to three anonymous reviewers for their constructive review comments. This study was supported in part by National Natural Science Foundation of China under Grants 41730960 and 41805040 and in part by the National Key R\&D Program of China under Grant 2017YFC1501602 and "the Fundamental Research Funds for the Central Universities"' under Grant 2019B02214. Y. Wang was supported in part by NSF Grant AGS-1834300.

\section{REFERENCES}

Bell, M. M., M. T. Montgomery, and W.-C. Lee, 2012: An axisymmetric view of concentric eyewall evolution in Hurricane Rita (2005). J. Atmos. Sci., 69, 2414-2432, https://doi.org/ 10.1175/JAS-D-11-0167.1.

Brand, S., 1970: Interaction of binary tropical cyclones of the western North Pacific Ocean. J. Appl. Meteor., 9, 433-441, https://doi.org/ 10.1175/1520-0450(1970)009<0433:IOBTCO > 2.0.CO;2.

Cha, D.-H., and Y. Wang, 2013: A dynamical initialization scheme for real-time forecasts of tropical cyclones using the WRF Model. Mon. Wea. Rev., 141, 964-986, https://doi.org/10.1175/ MWR-D-12-00077.1.

Chang, S. W., 1983: A numerical study of the interactions between two tropical cyclones. Mon. Wea. Rev., 111, 1806-1817, https://doi.org/ 10.1175/1520-0493(1983)111<1806:ANSOTI>2.0.CO;2.

Chen, S. Y. S., J. A. Knaff, and F. D. Marks, 2006: Effects of vertical wind shear and storm motion on tropical cyclone rainfall asymmetries deduced from TRMM. Mon. Wea. Rev., 134, 3190-3208, https://doi.org/10.1175/MWR3245.1.

Corbosiero, K. L., and J. Molinari, 2003: The relationship between storm motion, vertical wind shear, and convective asymmetries in tropical cyclones. J. Atmos. Sci., 60, 366-376, https://doi.org/ 10.1175/1520-0469(2003)060<0366:TRBSMV>2.0.CO;2.

DeHart, J. C., R. A. Houze Jr., and R. F. Rogers, 2014: Quadrant distribution of tropical cyclone inner-core kinematics in relation to environmental shear. J. Atmos. Sci., 71, 2713-2732, https://doi.org/10.1175/JAS-D-13-0298.1.

DeMaria, M., and J. C. L. Chan, 1984: Comments on "A numerical study of the interactions between two tropical cyclones." Mon. 
Wea. Rev., 112, 1643-1645, https://doi.org/10.1175/15200493(1984)112<1643:CONSOT>2.0.CO;2.

Donelan, M. A., B. K. Haus, N. Reul, W. J. Plant, M. Stiassnie, H. C. Graber, O. B. Brown, and E. S. Saltzman, 2004: On the limiting aerodynamic roughness of the ocean in very strong winds. Geophys. Res. Lett., 31, L18306, https://doi.org/10.1029/ 2004GL019460.

Dong, K. Q., and C. J. Neumann, 1983: On the relative motion of binary tropical cyclones. Mon. Wea. Rev., 111, 945-953, https://doi.org/ 10.1175/1520-0493(1983)111<0945:OTRMOB > 2.0.CO;2.

Dritschel, D. G., and D. W. Waugh, 1992: Quantification of the inelastic interaction of unequal vortices in two-dimensional vortex dynamics. Phys. Fluids, 4, 1737-1744, https://doi.org/ 10.1063/1.858394.

Falkovich, A. I., A. P. Khain, and I. Ginis, 1995: Motion and evolution of binary tropical cyclones in a coupled atmosphere-ocean numerical model. Mon. Wea. Rev., 123, 1345-1363, https://doi.org/ 10.1175/1520-0493(1995)123<1345:MAEOBT>2.0.CO;2.

Fiorino, M., and R. L. Elsberry, 1989: Some aspects of vortex structure related to tropical cyclone motion. J. Atmos. Sci., 46, 975-990, https://doi.org/10.1175/1520-0469(1989)046<0975: SAOVSR $>2.0 . \mathrm{CO} ; 2$.

Frank, W. M., and E. A. Ritchie, 2001: Effects of vertical wind shear on the intensity and structure of numerically simulated hurricanes. Mon. Wea. Rev., 129, 2249-2269, https://doi.org/ 10.1175/1520-0493(2001)129<2249:EOVWSO>2.0.CO;2.

Fu, H., Y. Wang, M. Riemer, and Q. Li, 2019: Effect of unidirectional vertical wind shear on tropical cyclone intensity change-Lowerlayer shear versus upper-layer shear. J. Geophys. Res. Atmos., 124, 6265-6282, https://doi.org/10.1029/2019JD030586.

Fujiwhara, S., 1921: The natural tendency towards symmetry of motion and its application as a principle in meteorology. Quart. J. Roy. Meteor. Soc., 47, 287-292, https://doi.org/ 10.1002/qj.49704720010.

- 1923: On the growth and decay of vortical systems. Quart. J. Roy. Meteor. Soc., 49, 75-104, https://doi.org/10.1002/ qj. 49704920602.

_ 1931: Short note on the behavior of two vortices. Proc. Phys. Math. Soc. Japan, 13, 106-110.

Gray, W. M., 1968: Global view of the origin of tropical disturbances and storms. Mon. Wea. Rev., 96, 669-700, https://doi.org/ 10.1175/1520-0493(1968)096<0669:GVOTOO >2.0.CO;2.

Gu, J.-F., Z.-M. Tan, and X. Qiu, 2015: Effects of vertical wind shear on inner core thermodynamics of an idealized simulated tropical cyclone. J. Atmos. Sci., 72, 511-530, https://doi.org/ 10.1175/JAS-D-14-0050.1.

— $-\ldots$, and —_, 2016: Quadrant-dependent evolution of low-level tangential wind of a tropical cyclone in the shear flow. J. Atmos. Sci., 73, 1159-1177, https://doi.org/10.1175/ JAS-D-15-0165.1.

Holland, G. J., and G. S. Dietachmayer, 1993: On the interaction of tropical-cyclone-scale vortices. III. Continuous barotropic vortices. Quart. J. Roy. Meteor. Soc., 119, 1381-1398, https:// doi.org/10.1002/qj.49711951408.

Hong, S. Y., J. Dudhia, and S. H. Chen, 2004: A revised approach to ice microphysical processes for the bulk parameterization of clouds and precipitation. Mon. Wea. Rev., 132, 103-120, https://doi.org/10.1175/1520-0493(2004)132<0103: ARATIM $>2.0 . \mathrm{CO} ; 2$.

—, Y. Noh, and J. Dudhia, 2006: A new vertical diffusion package with an explicit treatment of entrainment processes. Mon. Wea. Rev., 134, 2318-2341, https://doi.org/10.1175/ MWR3199.1.
Jang, W., and H. Y. Chun, 2013: The effects of topography on the evolution of Typhoon Saomai (2006) under the influence of Tropical Storm Bopha (2006). Mon. Wea. Rev., 141, 468-489, https://doi.org/10.1175/MWR-D-11-00241.1.

_- and ——, 2015a: Characteristics of binary tropical cyclones observed in the western North Pacific for 62 years (1951-2012). Mon. Wea. Rev., 143, 1749-1761, https:// doi.org/10.1175/MWR-D-14-00331.1.

$\longrightarrow$, and - 2015b: Effects of thermodynamic profiles on the interaction of binary tropical cyclones. J. Geophys. Res. Atmos., 120, 9173-9192, https://doi.org/10.1002/2015JD023409.

Jarrell, J., S. Brand, and D. S. Nicklin, 1978: An analysis of western North Pacific tropical cyclone forecast errors. Mon. Wea. Rev., 106, 925-937, https://doi.org/10.1175/1520-0493(1978)106<0925: AAOWNP $>2.0 . \mathrm{CO} ; 2$.

Jones, S. C., 1995: The evolution of vortices in vertical shear. I: Initially barotropic vortices. Quart. J. Roy. Meteor. Soc., 121, 821-851, https://doi.org/10.1002/qj.49712152406.

Jordan, C., 1958: Mean soundings for the West Indies area. J. Meteor., 15, 91-97, https://doi.org/10.1175/1520-0469(1958) 015<0091:MSFTWI > 2.0.CO;2.

Kain, J. S., and J. M. Fritsch, 1990: A one-dimensional entraining/ detraining plume model and its application in convective parameterization. J. Atmos. Sci., 47, 2784-2802, https://doi.org/ 10.1175/1520-0469(1990)047<2784:AODEPM>2.0.CO;2.

Khain, A., I. Ginis, A. Falkovich, and M. Frumin, 2000: Interaction of binary tropical cyclones in a coupled tropical cyclone-ocean model. J. Geophys. Res., 105, 22 337-22 354, https://doi.org/ 10.1029/2000JD900268.

Kuo, H.-C., G. T.-J. Chen, and C.-H. Lin, 2000: Merger of Tropical Cyclones Zeb and Alex. Mon. Wea. Rev., 128, 2967-2975, https://doi.org/10.1175/1520-0493(2000)128<2967: MOTCZA $>2.0 . C O ; 2$.

Lander, M., and G. J. Holland, 1993: On the interaction of tropicalcyclone-scale vortices. I: Observations. Quart. J. Roy. Meteor. Soc., 119, 1347-1361, https://doi.org/10.1002/qj.49711951406.

Liu, H.-Y., and Z.-M. Tan, 2016: A dynamical initialization scheme for binary tropical cyclones. Mon. Wea. Rev., 144, 4787-4803, https://doi.org/10.1175/MWR-D-16-0176.1.

Prieto, R., B. D. McNoldy, S. R. Fulton, and W. H. Schubert, 2003: A classification of binary tropical cyclone-like vortex interactions. Mon. Wea. Rev., 131, 2656-2666, https://doi.org/ 10.1175/1520-0493(2003)131<2656:ACOBTC > 2.0.CO;2.

Reasor, P. D., M. T. Montgomery, and L. D. Grasso, 2004: A new look at the problem of tropical cyclones in vertical shear flow: Vortex resiliency. J. Atmos. Sci., 61, 3-22, https://doi.org/ 10.1175/1520-0469(2004)061<0003:ANLATP>2.0.CO;2.

Ren, F., Y. Xie, B. Yin, M. Wang, and G. Li, 2020: Establishment of an objective standard for the definition of binary tropical cyclones in the western North Pacific. Adv. Atmos. Sci., 37, 1211-1221, https:// doi.org/10.1007/s00376-020-9287-3.

Ritchie, E. A., and G. J. Holland, 1993: On the interaction of tropical-cyclone-scale vortices. II: Discrete vortex patches. Quart. J. Roy. Meteor. Soc., 119, 1363-1379, https://doi.org/ 10.1002/qj.49711951407.

Shin, S.-E., J.-Y. Han, and J.-J. Baik, 2006: On the critical separation distance of binary vortices in a nondivergent barotropic atmosphere. J. Meteor. Soc. Japan, 84, 853-869, https://doi.org/ 10.2151/jmsj.84.853.

Simpson, R., and R. Riehl, 1958: Mid-tropospheric ventilation as a constraint on hurricane development and maintenance. Preprints, Tech. Conf. on Hurricanes, Miami Beach, FL, Amer. Meteor. Soc., D4-1-D4-10. 
Skamarock, W. C., and Coauthors, 2008: A description of the Advanced Research WRF version 3. NCAR Tech. Note NCAR/TN475+STR, 113 pp., https://doi.org/10.5065/D68S4MVH.

Stern, D. P., and F. Zhang, 2013: How does the eye warm? Part I: A potential temperature budget analysis of an idealized tropical cyclone. J. Atmos. Sci., 70, 73-90, https://doi.org/ 10.1175/JAS-D-11-0329.1.

Tang, B., and K. Emanuel, 2010: Midlevel ventilation's constraint on tropical cyclone intensity. J. Atmos. Sci., 67, 1817-1830, https://doi.org/10.1175/2010JAS3318.1.

Wang, Y., 2007: A multiply nested, movable mesh, fully compressible, nonhydrostatic tropical cyclone model-TCM4: Model description and development of asymmetries without explicit asymmetric forcing. Meteor. Atmos. Phys., 97, 93-116, https://doi.org/10.1007/s00703-006-0246-z.

_ 2008: Structure and formation of an annular hurricane simulated in a fully compressible, nonhydrostatic model-TCM4. J. Atmos. Sci., 65, 1505-1527, https://doi.org/10.1175/2007JAS2528.1.

_ Fujiwhara effect of binary cyclone (in Chinese). J. Acad. Meteor. Sci., 4, 14-20.

$\longrightarrow$, and,$- 1989 \mathrm{~b}$ : Interactions of binary vortices in a nondivergent barotropic model (in Chinese). J. Trop. Meteor., 5, 105-115, https://doi.org/10.16032/j.issn.1004-4965.1989.02.002.

_ scale vortices. IV: Baroclinic vortices. Quart. J. Roy. Meteor. Soc., 121, 95-126, https://doi.org/10.1002/qj.49712152106.

$\longrightarrow$, and — 1996: Tropical cyclone motion and evolution in vertical shear. J. Atmos. Sci., 53, 3313-3332, https://doi.org/ 10.1175/1520-0469(1996)053<3313:TCMAEI>2.0.CO;2.
Waugh, D. W., 1992: The efficiency of symmetric vortex merger. Phys. Fluids, 4, 1745-1758, https://doi.org/10.1063/1.858395.

Wu, C.-C., T.-S. Huang, W.-P. Huang, and K.-H. Chou, 2003: A new look at the binary interaction: Potential vorticity diagnosis of the unusual southward movement of Tropical Storm Bopha (2000) and its interaction with Supertyphoon Saomai (2000). Mon. Wea. Rev., 131, 1289-1300, https://doi.org/ 10.1175/1520-0493(2003)131<1289:ANLATB > 2.0.CO;2.

Wu, X., J.-F. Fei, X.-G. Huang, X.-P. Cheng, and J.-Q. Ren, 2011: Statistical classification and characteristics analysis of binary tropical cyclones over the western North Pacific Ocean. J. Trop. Meteor., 17, 335-344, https://doi.org/10.3969/ J.SSN.1006-8775.2011.04.003.

$\mathrm{Xu}$, Y., and Y. Wang, 2013: On the initial development of asymmetric vertical motion and horizontal relative flow in a mature tropical cyclone embedded in environmental vertical shear. J. Atmos. Sci., 70, 3471-3491, https://doi.org/10.1175/ JAS-D-12-0335.1.

Yang, C.-C., C.-C. Wu, K.-H. Chou, and C.-Y. Lee, 2008: Binary interaction between Typhoons Fengshen (2002) and Fungwong (2002) based on the potential vorticity diagnosis. Mon. Wea. Rev., 136, 4593-4611, https://doi.org/10.1175/2008MWR2496.1.

Zhang, D.-L., and H. Chen, 2012: Importance of the upper-level warm core in the rapid intensification of a tropical cyclone. Geophys. Res. Lett., 39, L02806, https://doi.org/10.1029/2011GL050578.

Zhang, J. A., R. F. Rogers, P. D. Reasor, E. W. Uhlhorn, and F. D. Marks Jr., 2013: Asymmetric hurricane boundary layer structure from dropsonde composites in relation to the environmental vertical wind shear. Mon. Wea. Rev., 141, 3968-3984, https://doi.org/10.1175/MWR-D-12-00335.1. 